\title{
UNIFORM ENERGY DISTRIBUTION IN A PATTERN-FORMING SYSTEM OF SURFACE CHARGES
}

\author{
KATARINA BELLOVA, ANTOINE JULIA, AND FELIX OTTO
}

\begin{abstract}
We consider a variational model for a charge density $u \in\{-1,1\}$ on a (hyper)plane, with a short-range attraction coming from the interfacial energy and a long-range repulsion coming from the electrostatic energy. This competition leads to pattern formation. We prove that the interfacial energy density is (asymptotically) equidistributed at scales large compared to the scale of the pattern. We follow the strategy laid out in 2]. The challenge comes from the reduced screening capabilities of surface charges compared to the volume charges considered in [2].
\end{abstract}

\section{Contents}

1. Introduction

2. The problem, notations and definitions

3. Statement of the main results

4. Uniform energy bound

5. Pointwise estimates on the fields

6. Boundary conditions are negligible in the thermodynamic limit.

7. Proofs

Acknowledgements

References

\section{INTRODUCTION}

The interplay of short-range attraction and long-range repulsion selects a length scale and typically leads to pattern formation. In a thermodynamic limit, provided the influence of boundary conditions fades away, this competition often seems to favor a periodic pattern, like equidistant stripes. Within variational models, periodicity has been established in 1-d situations, eg. [23], in 2-d models that are not too far from a packing problem, foremost [25] but also [6], or in models that feature a strong anisotropy, eg. [16].

Date: April 6, 2020.

2010 Mathematics Subject Classification. Primary : 49Q10; 35J50,49S05.

Key words and phrases. pattern formation, isoperimetric problem, elliptic regularity theory, longrange interactions, uniform energy distribution .

A.J. has been supported by University of Padova STARS Project "Sub-Riemannian Geometry and Geometric Measure Theory Issues: Old and New"; and by the INdAM - GNAMPA Project 2019 "Rectifiability in Carnot groups". 
In a multi-dimensional isotropic setting, even in a variational model, a proof of periodicity seems out of reach. However, robust strategies for proving a uniform distribution of eg. the energy density at scales large compared to the intrinsic scale are available, see [2]. That paper deals with the popular model where the configuration space consists of characteristic functions $u \in\{-1,1\}$, the short-range attractive interaction is the interfacial energy between the two phases, and the long-range repulsive interaction is electrostatic, with the order parameter $u$ playing the role of a charge density. While in [2], it is the volume fraction that is implicitly prescribed, the problem of prescribed volume is also of interest because of the potential loss of tightness, eg. [19, 20, and its isoperimetric aspects, eg. [10]; both problems are related in the regime of small volume fraction, eg. [9].

The main challenge in establishing a mesoscopically uniform energy distribution (which by a virial argument also leads to equipartition and thus uniform distribution of the interfacial energy) lies in capturing screening effects: On mesoscopic scales, charges arrange themselves in such a way as to reduce the macroscopic part of the electric field $\mathbf{b}$ as much as possible. In this paper, we consider a very similar problem, which however is of different dimensionality: While the field $\mathbf{b}$ extends into $d$-dimensional space $\mathbb{R}^{d}$, the charges $u$ are (hyper)surface charges constrained to $\mathbb{R}^{d-1} \times\{0\}$. In other words, we replace the squared $\dot{\mathrm{H}}^{-1}$-norm of $u$ by its fractional counterpart $\dot{\mathrm{H}}^{-\frac{1}{2}}$. This additional, dimensional, restriction of the charge density $|u| \leq 1$ clearly hinders screening and thus poses an additional challenge. In fact, it turns out that the arguments here, while following the same strategy, are more involved than in [2], as we shall detail below. In fact, some aspects are quite reminiscent of the deep work [12] on self-similarity of twin branching near an Austenite-Martensite interface. Incidentally, our setting of a thin (partial) conductor, leading to a field energy in form of a (squared) fractional Sobolev norm, resonates with the very active area of fractional elliptic equations and of thin obstacle problems; let us mention [8] as a popular reference.

The natural idea from statistical mechanics of establishing the negligibility of boundary effects by comparing different boundary conditions has been employed in [2], with the vanishing flux boundary condition being good for pasting, and the free boundary suitable for cutting. Incidentally, the ensuing monotonicity properties, which have been subsequently used for more subtle ferromagnetic pattern formation in [24, Lemmas 4 and 5], were also crucial in recent progress on quantitative stochastic homogenization [5]. Electrostatic screening also plays a role in the popular (mostly two-dimensional) models for Coulomb gases, and similar arguments have been used in this more subtle context, e.g. see the recent [4]. Let us also mention that the treatment of boundary layers with incomplete screening of [2] motivated a variational approach to the regularity of optimal transportation [18, see in particular Proposition 3.3 and Lemmas 2.3 and 2.4 therein.

We now give a short summary of this paper, contrasting it with [2]. We follow [2] in the sense that a first main step is an energy bound on mesoscopic scales (Theorem 3 here, Lemma 3.5 there). We also follow 2 in introducing a relaxed problem (meaning that the non-convex constraint $u \in\{-1,1\}$ is replaced by $u \in[-1,1]$ ), using its dual formulation (Lemma 8 here, Lemma 3.3 (a) there), appealing to a trace estimate (Lemma 9 here, Lemma 3.2 there), in order to obtain a non-linear estimate (Lemma 6 here, Lemma 3.3 (b) there). However, and this is the major complication, in this paper, even if we were 
to completely neglect the constraint on $u$, the field $\mathbf{b}$ still would not vanish. In fact, it leads to what we call the "over-relaxed problem". Hence the simple ODE argument that leads to Lemma 3.5 in [2] has to be replaced by a Campanato iteration (Lemma 3 ] here), adjusting "shifts" of the field at every scale (Lemma 2 using also Lemma 41). The similarities with [12, Theorem 2.1, (2.2)] "reverse bootstrap" are here.

As mentioned, we follow [2] in comparing free, no-flux, and periodic boundary conditions with their easy relations (Lemma 1 here, Lemma 3.1 there). Because of the fact that the relaxed problem (and even the over-relaxed problem) has a non-vanishing charge density, the pasting of a mesoscopically optimal pattern into the relaxed solution (Lemma 3.9 in [2]) is more involved. It requires an estimate of this over-relaxed charge density (Lemma 14 here, which is the main output of the regularity Section 5), and a finer modulation of the mesoscopically optimal pattern (based on Lemma 19, which is folklore). Only this yields Lemmas 16 and 17, which relate the mesoscopically localized energy to the one of the corresponding over-relaxed problem. By yet another Campanato-type iteration, both lemmas finally imply the negligibility of the energy of the over-relaxed problem (Lemma 18), and thus the main result (Theorem 11), from which derives the equipartition between the two contributions to the energy (Theorem 21).

The next sections contain the mathematical formulation of the problem (Section 2), the statements of the main theorems (Section 3), and three series of intermediate results on the way to Theorem 1 (Sections 4, 5, and 6). All the proofs are postponed to Section 7.

\section{The PRoblem, Notations And Definitions}

In the ambient space $\mathbb{R}^{d}$ with canonical basis $\left(\mathbf{e}_{1}, \ldots, \mathbf{e}_{d}\right)$, we consider two chemical species distributed on the hyperplane $\mathbb{R}^{d-1} \times\{0\}$. These species have different charge densities, renormalised here as +1 and -1 , respectively. The two different species also have a chemical interaction, we model this by introducing an energy term proportional to the interface area between them. In mathematical terms, the charge density is given by a function of locally bounded variation $u$ defined on the hyperplane and taking values \pm 1 . The interfacial energy is then proportional to the total variation of $u$ (the semi-norm defining the space BV, see Section 3.1 in [3]). The charges carried on the hyperplane generate an electric field $\mathbf{b}$ in the whole of $\mathbb{R}^{d}$, satisfying $\nabla \cdot \mathbf{b}=u \mathcal{H}^{d-1} \mathbf{L}\left(\mathbb{R}^{d-1} \times\{0\}\right)$ in the distributional sense. The electric energy is proportional to the square of the $\mathrm{L}^{2}$-norm of $\mathbf{b}$.

We work in a large cube $Q_{L}:=(-L / 2, L / 2)^{d-1} \times(0, L)$, the bottom face of which we denote by $\underline{Q_{L}}=\partial Q_{L} \cap\left\{x: x_{d}=0\right\}$. In the same way as in [2], we will study the minimizers $(u, \overline{\mathbf{b}}) \in \mathrm{BV}\left(\underline{Q_{L}}, \pm 1\right) \times \mathrm{L}^{2}\left(Q_{L}, \mathbb{R}^{d}\right)$ of the energy

$$
E\left(u, \mathbf{b}, Q_{L}\right):=\int_{\underline{Q_{L}}}|\nabla u|+\int_{Q_{L}} \frac{1}{2}|\mathbf{b}|^{2},
$$

under the following constraint, understood in the sense of distributions

$$
\left\{\begin{array}{l}
\mathbf{b} \cdot \mathbf{e}_{d}=u \text { on } \underline{Q_{L}}, \\
\nabla \cdot \mathbf{b}=0 \text { in } \overline{Q_{L}},
\end{array}\right.
$$


where, letting $\nu$ be the inner normal to the boundary, the normal component $\mathbf{b} \cdot \nu$ is well defined by application of the Divergence Theorem to vector fields $\zeta \mathbf{b}$, where $\zeta$ is a test function defined in the closure of $Q_{L}$. (See Section IX.2 of [13] for a description of spaces of divergence-free $L^{2}$ vector fields.) Omitting parameters in (1) means that length has been non-dimensionalized in such a way that the intrinsic scale of the pattern is unity. Energy has been non-dimensionalized so that the energy per $(d-1)$-dimensional area is of order one.

The problem could equivalently be formulated in $Q_{L} \cup\left(-Q_{L}\right)$, where the constraint on $Q_{L}$ could be formulated as a divergence equation in the distributional sense. This would be closer to the charge/field meaning of the pair $(u, \mathbf{b})$. However, by the symmetry of this problem, we consider only the upper cubel 1 As explained in the introduction, we seek a result on mesoscopically uniform energy density. To obtain this, we will study the minimizer on smaller cubes $Q_{l}$, and the lower parts of their boundaries $Q_{l}$. When studying a global minimizer on a smaller scale, we need to take into account the influence of the whole domain. This takes the form of flux (Neumann) boundary conditions imposed on the upper parts of the boundary of $Q_{L}: \Gamma_{L}:=\partial Q_{L} \backslash \underline{Q}_{L}$ (or $\Gamma_{l}$, for $Q_{l}$ ). We thus use various types of boundary conditions which we list here. Implicitly, we already considered the family of candidates with free boundary condition on $\Gamma_{L}$ :

$$
\mathcal{A}\left(Q_{L}\right):=\left\{(u, \mathbf{b}) \mid u \in \operatorname{BV}\left(\underline{Q_{L}}, \pm 1\right), \mathbf{b} \in \mathrm{L}^{2}\left(Q_{L}, \mathbb{R}^{d}\right),\left\{\begin{array}{l}
\nabla \cdot \mathbf{b}=0 \text { in } Q_{L}, \\
\mathbf{b} \cdot \nu=u \text { on } \underline{Q_{L}} .
\end{array}\right\}\right.
$$

We also consider subclasses of $\mathcal{A}\left(Q_{L}\right)$ corresponding to various flux boundary conditions on $\Gamma_{L}$ (again interpreted in the distributional sense). For $g \in \mathrm{L}^{2}\left(\Gamma_{L}\right)$, we will consider

$$
\mathcal{A}^{g}\left(Q_{L}\right):=\left\{(u, \mathbf{b}) \in \mathcal{A}\left(Q_{L}\right): \mathbf{b} \cdot \nu=g \text { on } \Gamma_{L}\right\} .
$$

Of course, $\mathcal{A}^{g}\left(Q_{L}\right)$ is only non-empty if $\left|\int_{\Gamma_{L}} g\right| \leq L^{d-1}$, so that both the divergence-free condition and the boundary condition $\mathbf{b} \cdot \nu= \pm 1$ on $\underline{Q}_{L}$ can be satisfied. A particular subclass which we will often study is the class of zero flux candidates

$$
\mathcal{A}^{0}\left(Q_{L}\right):=\left\{(u, \mathbf{b}) \in \mathcal{A}\left(Q_{L}\right): \mathbf{b} \cdot \nu=0 \text { on } \Gamma_{L}\right\} .
$$

Finally, it is particularly convenient to work in a horizontally periodic setting, as it is invariant under horizontal translations. The charge $u$ will then live in the torus $\mathbb{T}_{L}^{d-1}$ (the $(d-1)$ dimensional cube of side length $L$, identifying the opposite faces in the usual sense) and the field $\mathbf{b}$ in $\mathbb{T}_{L}^{d-1} \times(0, L)$. The boundary condition for $\mathbf{b}$ on the top face will be free in this case; and we have to define the energy slightly differently:

$$
E^{\mathrm{per}}\left(u, \mathbf{b}, Q_{L}\right):=\int_{\mathbb{T}_{L}^{d-1}}|\nabla u|+\int_{\mathbb{T}_{L}^{d-1} \times(0, L)} \frac{1}{2}|\mathbf{b}|^{2} .
$$

This notation suggests that we have identified $(u, \mathbf{b})$ on $\mathbb{T}_{L}^{d-1} \times(0, L)$ with horizontally periodic functions in $\mathbb{R}^{d-1} \times(0, L)$. In particular the divergence-free condition holds on the whole domain. However, we note that the energy takes into account (half of) the interface concentrated on $\partial\left(\underline{Q_{L}}\right)$.

\footnotetext{
${ }^{1}$ From a candidate in $Q_{L} \cup\left(-Q_{L}\right)$, one can define a symmetric candidate with no more energy by superposing the field $\mathbf{b} / 2$ and an appropriate reflection of $\mathbf{b} / 2$.
} 
We define the optimal energy densities corresponding to various classes candidates as

$$
\begin{aligned}
\sigma\left(Q_{L}\right) & :=\inf _{(u, \mathbf{b}) \in \mathcal{A}\left(Q_{L}\right)} \frac{E\left(u, \mathbf{b}, Q_{L}\right)}{L^{d-1}}, \\
\sigma^{0}\left(Q_{L}\right) & :=\inf _{(u, \mathbf{b}) \in \mathcal{A}^{0}\left(Q_{L}\right)} \frac{E\left(u, \mathbf{b}, Q_{L}\right)}{L^{d-1}}, \\
\sigma^{\text {per }}\left(Q_{L}\right) & :=\inf _{(u, \mathbf{b}) \in \mathcal{A}^{\text {per }}\left(Q_{L}\right)} \frac{E^{\text {per }}\left(u, \mathbf{b}, Q_{L}\right)}{L^{d-1}} .
\end{aligned}
$$

We note that, using the direct method of the calculus of variations, one can easily show that a minimizer of the functional (1) exists in each of the classes $\mathcal{A}\left(Q_{L}\right), \mathcal{A}^{\text {per }}\left(Q_{L}\right)$, $\mathcal{A}^{0}\left(Q_{L}\right)$ and $\mathcal{A}^{g}\left(Q_{L}\right)$ (for the latter, only if it is non empty).

We use the short-hand notation $\lesssim, \gtrsim$ for $\leq C$ and $\geq C$ with a constant $C \in(0,+\infty)$ depending only on the dimension $d, \sim$ stands for $\lesssim$ and $\gtrsim$ at the same time. Furthermore, a hypothesis of the form $H \ll 1$ for some quantity $H$, means that there exists a constant $C \in(0,+\infty)$ (still depending only on $d$ ) such that $H \leq C^{-1}$.

\section{Statement of the main Results}

The main results of this paper are the following two theorems:

Theorem 1 (Uniform distribution of energy). There exists a constant $\sigma^{*} \in(0,+\infty)$, depending only on $d$, such that for $L \gg 1$,

$$
\max \left\{\left|\sigma\left(Q_{L}\right)-\sigma^{*}\right|,\left|\sigma^{0}\left(Q_{L}\right)-\sigma^{*}\right|,\left|\sigma^{\text {per }}\left(Q_{L}\right)-\sigma^{*}\right|\right\} \lesssim \frac{1}{L^{1 / 2}}
$$

Furthermore, if $(u, \mathbf{b})$ is a minimizer in $\mathcal{A}^{\text {per }}\left(Q_{L}\right)$ and $L \geq l \gg 1$, then there holds

$$
\left|\frac{E\left(u, \mathbf{b}, Q_{l}\right)}{l^{d-1}}-\sigma^{*}\right| \lesssim \frac{1}{l^{1 / 2}} .
$$

We have no reason to believe that the exponent $1 / 2$ in (44) and (5) is optimal in any sense. However, it comes up naturally through Lemma 14, It should be compared to the (better) exponent 1 in the case of [2], which however can be improved by using the first variation (see Proposition 6.1 in that paper). We do not explore this direction in the present paper. A scaling argument similar to one used in [2, Theorem 1.2] yields:

Theorem 2 (Equipartition of the energy). For $(u, \mathbf{b})$ as above, if $L \geq l \gg 1$, then there hold

$$
\left|\frac{1}{l^{d-1}} \int_{\underline{Q_{l}}}\right| \nabla u\left|-\frac{\sigma^{*}}{2}\right| \lesssim \frac{1}{l^{1 / 4}} \quad \text { and }\left.\quad\left|\frac{1}{l^{d-1}} \int_{Q_{l}} \frac{1}{2}\right| \mathbf{b}\right|^{2}-\frac{\sigma^{*}}{2} \mid \lesssim \frac{1}{l^{1 / 4}} .
$$

\section{UNIFORM ENERGY BOUND}

As in [2], the first main step in establishing Theorems 1 and 2 is a uniform bound on the local energy density:

Theorem 3 (Uniform bound on the energy density). Let $(u, \mathbf{b})$ be a minimizer of (1) in the class $\mathcal{A}^{\text {per }}\left(Q_{L}\right)$ with $L \gg 1$. Then given l with $L \geq l \gg 1$, there holds

$$
\frac{E\left(u, \mathbf{b}, Q_{l}\right)}{l^{d-1}} \lesssim 1
$$


(Since we formulate Theorem 3 in terms of the periodic problem, there is no loss of generality in considering centered cubes $Q_{l}$.) Clearly, the task at hand is to pass the global energy estimate down to a local one, which will be done iteratively. The global energy estimate is a consequence of the following easy lemma. It collects all the obvious relations, including the natural monotonicities which follow from cutting and pasting, and some easy estimates on the various global energy densities.

Lemma 1 (Basic inequalities). There exists a constant $C \in(0,+\infty)$, depending only on $d$, such that for $L \gg 1$ :

(i) $\sigma\left(Q_{L}\right) \leq \sigma^{\text {per }}\left(Q_{L}\right)$ and $\sigma\left(Q_{L}\right) \leq \sigma^{0}\left(Q_{L}\right)$,

(ii) $\sigma\left(Q_{L}\right) \leq \sigma\left(Q_{k L}\right)$ for each positive integer $k$,

(iii) $\sigma^{0}\left(Q_{k L}\right) \leq \sigma^{0}\left(Q_{L}\right)$ for each positive integer $k$,

(iv) $\sigma^{0}\left(Q_{L}\right) \leq C$,

(v) $\sigma\left(Q_{L}\right) \geq 1 / C$

(vi) $\sigma^{\text {per }}\left(Q_{L}\right) \leq \sigma\left(Q_{L}\right)+C / L$.

As in [2], the main challenge in establishing Theorem 3 consists in controlling the long-range interaction via the field. This relies on screening in the sense of electrostatics, i. e. the reduction of the size of the field $\mathbf{b}$ through a rearrangement of the charges $u$. More precisely, the issue is how effective this screening is in the presence of some flux boundary data $g$ on $\Gamma_{L}$. If the charges $u$ were not constrained at all, we would arrive at the following "over-relaxed" problem:

$$
E_{0}^{g}\left(Q_{L}\right):=\inf \left\{\int_{Q_{L}} \frac{1}{2}|\mathbf{b}|^{2} \mid \begin{array}{l}
\nabla \cdot \mathbf{b}=0 \text { in } Q_{L} \\
\mathbf{b} \cdot \nu=g \text { on } \Gamma_{L}
\end{array}\right\}
$$

It is standard that a divergence-free field minimizing this energy is the gradient of a potential $\mathbf{b}=-\nabla v_{0}$ (see Section IX.3 of [13]). Furthermore, because of the free boundary conditions on $Q_{L}$, the first variation of the field energy yields that $\mathbf{b}$ is $\mathrm{L}^{2}$-orthogonal to all divergence-free vector fields $\tilde{\mathbf{b}}$ (not necessarily vanishing on $Q_{L}$ ). By integration by parts, this implies the vanishing of the boundary integral of $v_{0} \tilde{\mathbf{b}} \cdot \nu$. Since any flux boundary data $g$ of vanishing boundary integral can be extended to a divergence-free field $\tilde{\mathbf{b}}$, this implies that the trace of $v_{0}$ is orthogonal to all functions $g$ of vanishing boundary integral, and thus has to be constant. Therefore $v_{0}$ can be chosen as the solution to

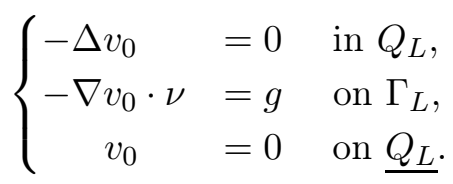

Even in this ideal situation, screening is incomplete: While reducing the horizontal components of the field near the surface $\underline{Q}_{L}$, it has little effect on the vertical component. The main insight is that, on large scales, the non-relaxed problem essentially has the same decay properties as the over-relaxed one when passing from $Q_{l}$ to the smaller $Q_{\theta l}$; this is the content of the following lemma. We consider the quantity $F(\beta, l)$, corresponding to the volume-averaged energy of a fixed pair $(u, \mathbf{b})$, at scale $l$ after a vertical shift $\beta$ :

$$
F(\beta, l):=\frac{E\left(u, \mathbf{b}-\beta \mathbf{e}_{d}, Q_{l}\right)}{l^{d}} .
$$


Lemma 2 (One-step improvement). Let $(\mathbf{b}, u)$ be a minimizer in $\mathcal{A}^{\text {per }}\left(Q_{L}\right)$. There exist constants $\delta \in(0,+\infty)$ and $\theta \in(0,1 / 2]$, depending only on $d$, such that the following holds. If $L \geq l \gg 1$ and

$$
\beta \in[-1 / 2,1 / 2] \quad \text { is such that } F(\beta, l) \leq \delta,
$$

then there exists a new shift $\tilde{\beta}$ such that

$$
|\beta-\tilde{\beta}| \lesssim F(\beta, l)^{1 / 2} \quad \text { and } \quad F(\tilde{\beta}, \theta l)-\theta F(\beta, l) \lesssim \frac{1}{l} .
$$

In regularity theory such a result is known as a one-step improvement lemma in a Campanato iteration. As usual in Campanato's characterization of Hölder spaces, the (squared) volume average $F$ involves constant shifts, which in view of our comments after (6) reduces to the vertical component (and thus is parameterized by a scalar $\beta$ ). As usual in this theory, Lemma 2 feeds into a Campanato iteration, of which we just retain how the error term $\mathrm{Cl}^{-1}$ in (9) affects the bound on small scales, which thanks to the volume average in $F$ is still finer information than needed for the area average in Theorem 3 ,

Lemma 3 (Campanato iteration). Let $(\mathbf{b}, u)$ be minimizing in $\mathcal{A}^{\text {per }}\left(Q_{L}\right)$. There exists a constant $\delta \in(0,+\infty)$, depending only on $d$, such that the following holds: If $F(0, L) \leq \delta$ and $L \geq l \gg 1$, then there holds

$$
F(0, l) \lesssim F(0, L)+\frac{1}{l} .
$$

We now explain the route towards Lemma 22. Note that (9) could be strengthened to $F(\tilde{\beta}, \theta l)-\theta^{\alpha} F(\beta, l) \leq C_{\alpha} l^{-1}$ for any $\alpha \in(0,2)$. In fact, the simpler $F(\tilde{\beta}, \theta l) \leq \theta^{\alpha} F(\beta, l)$ would be obvious on the level of the over-relaxed problem. The main work consists in appealing to local optimality for $(\mathbf{b}, u)$ in order to lift this to the non-convex problem, at the expense of the error term $\mathrm{Cl}^{-1}$. Following [2], this is done via the convex "relaxed" problem, which in this paper plays an intermediate role between non-relaxed and overrelaxed problem (and is just needed in this section):

$$
E_{r e l}^{g}\left(Q_{L}\right):=\inf \left\{\int_{Q_{L}} \frac{1}{2}|\mathbf{b}|^{2} \mid \begin{array}{ccc}
\nabla \cdot \mathbf{b} & =0 & \text { in } Q_{L}, \\
\mathbf{b} \cdot \nu & \in[-1,1] & \text { on } \frac{Q_{L}}{\mathbf{b}_{L} \cdot \nu} \\
\mathbf{b} \cdot \nu & \text { on } \Gamma_{L}
\end{array}\right\}
$$

In Lemma 4, we show that indeed the over-relaxed problem is close to the relaxed problem; and in Lemma 5, we establish that the relaxed problem is close to the original one in terms of energy.

Lemma 4 (Closeness of over-relaxed to relaxed problem). Let $\mathbf{b}$ be a solution to the relaxed problem (10) and $\beta \in[-1 / 2,1 / 2]$ be fixed. Let $-\nabla v_{0}$ be the solution to the over-relaxed problem (6) with flux boundary data replaced by $\left(\mathbf{b}-\beta \mathbf{e}_{d}\right) \cdot \nu$ on $\Gamma_{L}$. Then, supposing $\int_{\Gamma_{L}}\left(\left(\mathbf{b}-\beta \mathbf{e}_{d}\right) \cdot \nu\right)^{2} \ll L^{d-1}$, there holds

$$
\int_{Q_{L}}\left|\mathbf{b}-\beta \mathbf{e}_{d}+\nabla v_{0}\right|^{2} \lesssim\left(\int_{\Gamma_{L}}\left(\left(\mathbf{b}-\beta \mathbf{e}_{d}\right) \cdot \nu\right)^{2}\right)^{\frac{d}{d-1}} .
$$


Lemma 5 (Closeness of relaxed to original problem). Given a minimizer $\overline{\mathbf{b}}$ for the relaxed problem (10), there exist $(\mathbf{b}, u) \in \mathcal{A}^{g}\left(Q_{L}\right)$ such that

$$
\int_{\underline{Q_{L}}}|\nabla u|+\int_{Q_{L}} \frac{1}{2}|\mathbf{b}-\overline{\mathbf{b}}|^{2} \lesssim L^{d-1} .
$$

While the proof of Lemma 5 is a straightforward post-processing, which relies on elementary elliptic estimates in form of Lemma 11 below, Lemma 4 is more subtle. In fact, we first establish a version of Lemma 4 without the shift.

Lemma 6 (Nonlinear estimate). Given $g$ with $\int_{\Gamma_{L}} g^{2} \ll L^{d-1}$, if $\mathbf{b}$ is a minimizer for the relaxed problem (10), and $-\nabla v_{0}$ is the minimizer of the over-relaxed problem (6), then there holds

$$
\int_{Q_{L}} \frac{1}{2}\left|\mathbf{b}+\nabla v_{0}\right|^{2} \lesssim\left(\int_{\Gamma_{L}} g^{2}\right)^{\frac{d}{d-1}}
$$

Here, as in Lemma 4 , the crucial aspect of this non-linear estimate is that the exponent $d /(d-1)$ appearing on the r. h. s. is (strictly) larger than one. Both Lemma 6 and 4 crucially rely on an obvious $\mathrm{L}^{2}$-orthogonality between the over-relaxed problem and the two others, which we state for drama.

Lemma 7 (Orthogonality). Let $\mathbf{b}$ be a divergence-free field and $v_{0}$ be the solution to the over-relaxed problem (6) in $Q_{L}$ with $g=\mathbf{b} \cdot \nu$ on $\Gamma_{L}$. Then there holds

$$
\int_{Q_{L}} \frac{1}{2}\left|\mathbf{b}+\nabla v_{0}\right|^{2}=\int_{Q_{L}} \frac{1}{2}|\mathbf{b}|^{2}-\int_{Q_{L}} \frac{1}{2}\left|\nabla v_{0}\right|^{2} .
$$

Modulo Lemma 7, we follow the approach of [2] to establish Lemma 6: We appeal to a dual formulation of the convex relaxed problem (Lemma 8), which reduces Lemma 6 , to a trace estimate (Lemma 9).

Lemma 8 (Dual to the relaxed problem). Given $g \in \mathrm{L}^{2}\left(\Gamma_{L}\right)$ with $\left|\int_{\Gamma_{L}} g\right| \leq L^{d-1}$ there holds

$$
E_{r e l}^{g}\left(Q_{L}\right)=-\inf \left\{\int_{Q_{L}} \frac{1}{2}|\nabla v|^{2}+\int_{\underline{Q_{L}}}|v|-\int_{\Gamma_{L}} v g \mid v \in \mathrm{H}^{1}\left(Q_{L}\right)\right\} .
$$

Lemma 9 (Trace estimate). Given a function $w \in \mathrm{H}^{1}\left(Q_{L}\right)$, then for any $\epsilon \in(0,1]$ there holds

$$
\left(\int_{\underline{Q_{L}}} w^{2}\right)^{\frac{1}{2}} \lesssim(\epsilon L)^{\frac{1}{2}}\left(\int_{Q_{L}}|\nabla w|^{2}\right)^{\frac{1}{2}}+\frac{1}{(\epsilon L)^{\frac{d-1}{2}}} \int_{\underline{Q_{L}}}|w| .
$$

We conclude this section by stating two elliptic estimates that we need. We could not find a reference for the maximal regularity expressed in Lemma 10 and thus provide a proof. Lemma 11 is standard and we include its elementary proof for convenience.

Lemma 10 (Normal flux estimate). For any harmonic function $v$ on the cube $[0, L]^{d}$ which vanishes on its bottom face $x_{d}=0$ we have for the normal derivative $\partial_{\nu} v$ on the boundary $\partial(0, L)^{d}$ :

$$
\int_{\left\{x_{d}=0\right\}}\left(\partial_{\nu} v\right)^{2} \lesssim \int_{\Gamma}\left(\partial_{\nu} v\right)^{2},
$$


where $\Gamma:=\left(\partial(0, L)^{d}\right) \backslash\left\{x_{d}=0\right\}$.

Lemma 11 (Harmonic building block). Let $g$ be such that $\int_{\partial Q_{L}} g=0$ and solve

$$
\left\{\begin{array}{lll}
\Delta v & =0 & \text { in } Q_{L} \\
\nabla v \cdot \nu & =g & \text { on } \partial Q_{L}
\end{array}\right.
$$

Then for $p \in[2(d-1) / d, 2]$ there holds

$$
\int_{Q_{L}}|\nabla v|^{2} \lesssim L^{d-(d-1) \frac{2}{p}}\left(\int_{\partial Q_{L}}|g|^{p}\right)^{\frac{2}{p}} .
$$

\section{Pointwise estimates on the fields}

This technical section is devoted to pointwise bounds on the field $\mathbf{b}$ coming from a fixed energy minimizer $(u, \mathbf{b})$ in $\mathcal{A}^{\text {per }}\left(Q_{L}\right)$. Rather than the field $\mathbf{b}$, we are interested in its potential $v$, which in fact is also horizontally periodic:

Lemma 12 (Periodicity of the potential $v$ ). There exists $v$ such that $\mathbf{b}=-\nabla v, v$ is periodic in the horizontal directions and $v=0$ on $\left\{x_{d}=L\right\}$.

We think of $v$ and $\mathbf{b}$ as fields on $\mathbb{R}^{d-1} \times(0, L)$ that are horizontally periodic; we are also interested in the corresponding (non-periodic) over-relaxed problem $E_{0}^{\mathbf{b} \cdot \nu}\left(Q_{l}\right)$ on $Q_{l}$ with flux boundary data given by $b \cdot \nu$ on $\Gamma_{l}$, cf. (6), for some $l \leq L$. The upcoming pointwise bounds are on both potentials $v$ and $v_{0}$, which share the same flux boundary data on $\Gamma_{l}$, as well as on the charge density $-\partial_{d} v_{0}$ on $\underline{Q}_{l}$ coming from the over-relaxed potential:

Lemma 13 (Pointwise bound on the potentials). The potential $v$ and $v_{0}$ are uniformly Hölder- $1 / 2$ continuous, i.e. letting $[\cdot]_{\mathrm{C}^{1 / 2}(Q)}$ stand for the Hölder-1/2 semi-norm on the cube $Q$, there holds

$$
[v]_{\mathrm{C}^{1 / 2}\left(Q_{L}\right)} \lesssim 1 \quad \text { and } \quad\left[v_{0}\right]_{\mathrm{C}^{1 / 2}\left(Q_{l}\right)} \lesssim 1
$$

Lemma 14 (Pointwise bound on the over-relaxed charge density). Let $Q_{l}$ and $v_{0}$ be as in the previous lemma. Then there holds

$$
\left|\partial_{d} v_{0}\left(x^{\prime}, 0\right)\right| \lesssim \operatorname{dist}\left(x^{\prime}, \Gamma_{l}\right)^{-\frac{1}{2}} \quad \text { for all }\left(x^{\prime}, 0\right) \in \underline{Q_{l}} .
$$

These two results will be crucially used in the constructions of Section 6, they just rely on the a priori bound of Theorem 3 and follow from elementary potential theory. To be more precise, Lemma 14 is a straightforward consequence of the $v_{0}$-part of Lemma 13, which in view of the relation between $v_{0}$ and $v$ easily follows from the $v$-part of Lemma 13. The $v$-part of Lemma 13 is an easy consequence of the following two pointwise bounds on $\mathbf{b}=-\nabla v$ :

Corollary 1 (Pointwise bound on the field away from the surface). Given $\left(x^{\prime}, x_{d}\right) \in Q_{L}$ with $1 \ll x_{d} \leq L$, there holds

$$
\left|\mathbf{b}\left(x^{\prime}, x_{d}\right)\right| \lesssim x_{d}^{-\frac{1}{2}}
$$


Lemma 15 (Pointwise bound on the field near the surface). Given $\left(x^{\prime}, x_{d}\right) \in Q_{L}$ with $x_{d} \ll 1$, there holds

$$
\left|\mathbf{b}\left(x^{\prime}, x_{d}\right)\right| \lesssim \ln \left(\frac{1}{x_{d}}\right) .
$$

The bound of Corollary 1 is an immediate consequence of Theorem 3 , whereas the bound of Lemma 15, which we only use to extend the bound of Corollary 1 to the range $x_{d} \ll 1$, follows by elementary potential theory from the boundedness of the charge density.

\section{Boundary CONDITIONS ARE NEGLigible in the THERMOdYNAMIC Limit.}

This section leads to Theorem 1 via a series of lemmas. Throughout the section, $(u, \mathbf{b})$ denotes a minimizer in $\mathcal{A}^{\text {per }}\left(Q_{L}\right)$ with $L \gg 1$. The main strategy for the proof of Theorem 1 is to relate the local energy $E\left(u, \mathbf{b}, Q_{l}\right)$ (always in the regime $l \gg 1$ ) to the sum of $\sigma^{0}\left(Q_{l}\right)$ (or $\sigma^{p e r}\left(Q_{l}\right)$ ) and the energy $\int_{Q_{l}} \frac{1}{2}\left|\nabla v_{0}\right|^{2}$ of the solution $v_{0}$ to the over-relaxed problem (6) on $Q_{l}$ with flux boundary data $\mathbf{b} \cdot \nu$ on $\Gamma_{l}$. On the level of the lower bound, this is done in Lemma 16, for the upper bound, it is Lemma 17.

Lemma 16 (Precise lower bound on the local energy). There exists a constant $C$, depending only on $d$, such that if $L \geq l \gg 1$, then there holds

$$
\frac{1}{l^{d-1}}\left(E\left(u, \mathbf{b}, Q_{l}\right)-\int_{Q_{l}} \frac{1}{2}\left|\nabla v_{0}\right|^{2}\right) \geq \sigma^{0}\left(Q_{l}\right)-\frac{C}{l^{1 / 2}} .
$$

Lemma 17 (Precise upper bound on the local energy). There exists a constant $C$, depending only on $d$, such that if $L \geq l \gg 1$, then there holds

$$
\frac{1}{l^{d-1}}\left(E\left(u, \mathbf{b}, Q_{l}\right)-\int_{Q_{l}} \frac{1}{2}\left|\nabla v_{0}\right|^{2}\right) \leq \sigma^{p e r}\left(Q_{l}\right)+\frac{C}{l^{1 / 2}} .
$$

Of course, both lemmas are established by constructions: In Lemma 16 we construct a candidate for $\mathcal{A}^{0}\left(Q_{l}\right)$ based on $\left(u, \mathbf{b}+\nabla v_{0}\right)$, which by definition of $-\nabla v_{0}$ has vanishing flux boundary data, while Lemma 17 uses a minimizer $\left(u^{\text {per }}, \mathbf{b}^{\text {per }}\right)$ in $\mathcal{A}^{\text {per }}\left(Q_{l}\right)$ to construct a competitor for $(u, \mathbf{b})$ in $Q_{l}$ based on $\left(u^{\text {per }}, \mathbf{b}^{\text {per }}+\nabla v_{0}^{p e r}-\nabla v_{0}\right)$, which has the right boundary conditions if $v_{0}^{p e r}$ is the solution to the over-relaxed problem (6) with flux boundary data $g:=\mathbf{b}^{\text {per }} \cdot \nu$ on $\Gamma_{l}$. Loosely speaking, both lemmas express an orthogonality between the micro-structured part and the over-relaxed part, which is again based on Lemma 7. Both lemmas express this relationship on the level of a relative error decaying as $l^{-1 / 2}$. An easy consequence of Lemma 16 for $l=L$ and of Lemma 1 is the first part of Theorem 1 in the form of

Corollary 2 (Boundary conditions do not affect the global energy density). The limit

$$
\sigma^{*}=\lim _{L \rightarrow \infty} \sigma\left(Q_{L}\right)=\lim _{L \rightarrow \infty} \sigma^{0}\left(Q_{L}\right)=\lim _{L \rightarrow \infty} \sigma^{p e r}\left(Q_{L}\right)
$$

exists, with the convergence rate

$$
\max \left\{\left|\sigma\left(Q_{L}\right)-\sigma^{*}\right|,\left|\sigma^{0}\left(Q_{L}\right)-\sigma^{*}\right|,\left|\sigma^{p e r}\left(Q_{L}\right)-\sigma^{*}\right|\right\} \lesssim \frac{1}{L^{1 / 2}} .
$$

The second part of Theorem 1 follows from Lemmas 16 and 17 once we establish that the energy of the over-relaxed problem is negligible: 
Lemma 18 (Over-relaxed solutions are energetically negligible). Given $L \gg 1$ and $\theta \ll 1$ such that $\theta^{-1} \in 2 \mathbb{N}+1$, if $l \gg 1$ is of the form $l=\theta^{N} L$ for some positive integer $N$ and $v_{0}$ is the solution to the over-relaxed problem (6) induced by $\mathbf{b}$ on $Q_{l}$, then there holds

$$
\frac{1}{l^{d-1}} \int_{Q_{l}} \frac{1}{2}\left|\nabla v_{0}\right|^{2} \lesssim \frac{1}{l^{1 / 2}}
$$

While not very technical, Lemma 18 is subtle: The combination of Lemmas 16 and 17 (in conjunction with Corollary 21) allows us to relate the over-relaxed solution of a big cube to those of smaller cubes that partition the big one. This drives yet another Campanato iteration based on the (boundary) regularity of the over-relaxed problem, akin to Lemmas 2 and 3 .

The constructions of Lemmas [16 and 17] are technical: In the case of Lemma 16, the pair $\left(u, \mathbf{b}+\nabla v_{0}\right)$ is not admissible in $\mathcal{A}^{0}\left(Q_{l}\right)$ due to the additional charge $-\partial_{d} v_{0}$ on $\underline{Q}_{l}$. Thanks to Lemma 14, this extra charge is small outside of a boundary layer. In order to achieve the related scaling $l^{-1 / 2}$ of the relative error, we adjust for this small amount of extra charge by a smooth deformation of the set described by the characteristic function $u$. This type of deformation of $u \in\{-1,1\}$ into another characteristic function $\tilde{u}$ in order to change the volume (fraction) while controlling the surface area is folklore. We did not find in the literature a suitable statement for the quantitative modification of a set of finite perimeter, though one might try and start from the perturbation defined in [14]. We state (and prove) what we need for the convenience of the reader:

Lemma 19 (Smooth deformation of sets to adjust their volume). Let $\lambda \geq 1$ and $\underline{Q}=$ $(0, \lambda)^{d-1}$ be a $(d-1)$-dimensional cube. Consider a function $u=\{1,-1\}$ on $\underline{Q}$ with

$$
\begin{gathered}
\left|f_{\underline{Q}} u\right| \leq \frac{1}{2}, \\
\int_{\underline{Q}}|\nabla u| \leq \lambda^{d-2} \Lambda,
\end{gathered}
$$

for some $\Lambda<+\infty$. Then there exists a constant $m_{0} \in(0,+\infty)$, depending only on $\Lambda$ and $d$, such that for any $m \in\left[-m_{0}, m_{0}\right]$ there exists $\tilde{u}=\{-1,1\}$ with $(u-\tilde{u})$ compactly supported in $\underline{Q}$ and

$$
\begin{aligned}
& f_{\underline{Q}} \tilde{u}=f_{\underline{Q}} u+m, \quad f_{\underline{Q}}|\tilde{u}-u| \lesssim \Lambda|m|, \\
& \int_{\underline{Q}}|\nabla \tilde{u}|-\int_{\underline{Q}}|\nabla u| \lesssim \Lambda \lambda^{d-2}|m|,
\end{aligned}
$$

where the implicit constants in the last two inequalities depend only on $\Lambda$ and $d$.

Our application of Lemma 19 relies on the fact that we have "enough room" to perturb the local volume fraction. This is a consequence of Theorem 3, which ensures that the charge distribution $u$ is approximately neutral on large scales: 
Lemma 20 (Neutrality on large-scale averages). If $(u, \mathbf{b})$ is a minimizer in $\mathcal{A}^{\text {per }}\left(Q_{l}\right)$ and $l \geq \lambda \gg 1$, then there holds

$$
\left|f_{\underline{Q}_{\lambda}} u\right| \leq \frac{1}{2} .
$$

\section{Proofs}

In this section, we provide the proofs of the theorems, lemmas, and corollaries in the order they were stated.

Proof of Theorem 1. The convergence of $\sigma\left(Q_{L}\right), \sigma^{\text {per }}\left(Q_{L}\right)$, and $\sigma^{0}\left(Q_{L}\right)$ to $\sigma^{*}$ as $L \uparrow+\infty$, is established in Corollary 2, We turn to (5). We start by considering a cube $Q_{l}$ contained in $Q_{L}$ with $l=\theta^{n} L$ for some integer $n$ and some $\theta \sim 1$ with $\theta^{-1} \in 2 \mathbb{N}+1$ such that Lemma 18 holds. By Lemma 16, Lemma 17, and (4) at scale $l$, there holds

$$
\left.\left|\frac{E\left(u, \mathbf{b}, Q_{l}\right)}{l^{d-1}}-\frac{1}{l^{d-1}} \int_{Q_{l}} \frac{1}{2}\right| \nabla v_{0}\right|^{2}-\sigma^{*} \mid \lesssim \frac{1}{l^{1 / 2}} .
$$

By Lemma 18 the term involving $\nabla v_{0}$ is controlled by $C l^{-1 / 2}$ and we may conclude for such cubes.

So far, we have proved (5) for boxes of lateral size $l$ of the form $\theta^{n} L, n \in \mathbb{N}$, where $\theta^{-1} \in 2 \mathbb{N}+1$ with $\theta \sim 1$ coming from Lemma 18. We notice that Lemma [16] and Corollary 2 imply

$$
E(u, \mathbf{b}, Q)-\sigma^{*}|\underline{Q}| \gtrsim-l^{d-3 / 2}
$$

for all boxes $Q$ of size $l \leq L$ (the statement is trivial for $l \ll 1$ ). We note that the l. h. s. $E(u, \mathbf{b}, C)-\sigma^{*}|\underline{C}|$ is super-additive in sets $C$ touching the bottom. Since any $l \in[0, L)$ can be written as linear combination of $\left\{2^{-n} L\right\}_{n \in \mathbb{N}}$ with coefficients in $\{0,1\}$, and since $d-3 / 2>0$ we obtain that (15) holds for any non-square boxes $C$ of lateral size smaller than $l$, too. For a given box $Q$, there exists a box $Q^{\prime}$ of side-length of the form $\theta^{n} L$ and $d$ non-square boxes $C$ such that $\underline{Q}^{\prime}$ is the (disjoint) union of $\underline{Q}$ and the $\underline{C}$ 's. Hence by the above-mentioned super-additivity, and by (15), we may lift the opposite estimate

$$
E(u, \mathbf{b}, Q)-\sigma^{*}|\underline{Q}| \lesssim l^{d-3 / 2}
$$

from $Q^{\prime}$ to $Q$.

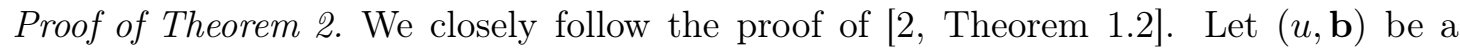
minimizer in $\mathcal{A}^{\text {per }}\left(Q_{L}\right)$, by Theorem 1 there exists $C \in(0,+\infty)$ such that if $L \geq l \gg 1$, then there holds

$$
\frac{E\left(u, \mathbf{b}, Q_{l}\right)}{l^{d-1}} \leq \sigma^{*}+\frac{C}{l^{1 / 2}} .
$$

On the other hand, for $\lambda \in[1 / 2,3 / 2]$ the rescaled pair $\left(u^{\lambda}, \mathbf{b}^{\lambda}\right)$ defined by

$$
u^{\lambda}(\lambda x)=u(x) \quad \text { and } \quad \mathbf{b}^{\lambda}(\lambda x)=\mathbf{b}(x)
$$


is a candidate in $\mathcal{A}^{\text {per }}\left(Q_{\lambda L}\right)$. In particular, the restriction of $\left(u^{\lambda}, \mathbf{b}^{\lambda}\right)$ to $Q_{\lambda l}$ is a candidate in $\mathcal{A}\left(Q_{\lambda l}\right)$. Using this and (4) in Theorem 1 at scale $\lambda l$, we get

$$
f(\lambda):=\frac{E\left(u^{\lambda}, \mathbf{b}^{\lambda}, Q_{\lambda l}\right)}{(\lambda l)^{d-1}} \geq \sigma\left(Q_{\lambda l}\right) \geq \sigma^{*}-\frac{C}{(\lambda l)^{1 / 2}} .
$$

In this notation, the combination of (16) and (17) yields

$$
f(\lambda)-f(1) \gtrsim-\frac{1}{l^{1 / 2}} \text { and } f(1) \lesssim 1 .
$$

It follows from a change of variables that $f$ is of the form

$$
f(\lambda)=\frac{1}{\lambda} a+\lambda b \quad \text { with } a:=\frac{1}{l^{d-1}} \int_{\underline{Q_{l}}}|\nabla u|, b:=\frac{1}{l^{d-1}} \int_{Q_{l}} \frac{1}{2}|\mathbf{b}|^{2} .
$$

Since $f^{\prime \prime}(\lambda)=2 a / \lambda^{3} \leq 16 f(1)$, it follows from the second item in (18) that $\left|f^{\prime \prime}(\lambda)\right| \lesssim 1$, so that by Taylor's formula for $t \in[-1 / 2,1 / 2]$,

$$
\left|f^{\prime}(1)-\frac{1}{t}(f(1+t)-f(1))\right| \lesssim|t| .
$$

Thus, for $t= \pm 1 / l^{1 / 4}$ and by the first item in (18) this implies $\left|f^{\prime}(1)\right| \lesssim 1 / l^{1 / 4}$, which because of $f^{\prime}(1)=b-a$, see (19), yields the claim of the theorem.

Proof of Theorem 3. Let $(\mathbf{b}, u)$ be minimizing in $\mathcal{A}^{\text {per }}\left(Q_{L}\right)$. Provided $L \gg 1$, by statements (vi), (ii) and (iv) of Lemma 1, and in view of definition (3), we have

$$
\frac{E\left(u, \mathbf{b}, Q_{L}\right)}{L^{d-1}} \lesssim 1
$$

By definition (77) again provided $L \gg 1$, we thus have $F(0, L) \leq C / L \leq \delta$, where $\delta$ is as in Lemma 3. Applying this Lemma for $L \geq l \gg 1$, we get

$$
E\left(u, \mathbf{b}, Q_{l}\right) \lesssim \frac{l^{d}}{L^{d}} E\left(u, \mathbf{b}, Q_{L}\right)+l^{d-1}=l^{d-1}\left(\frac{l}{L} \frac{E\left(u, \mathbf{b}, Q_{L}\right)}{L^{d-1}}+1\right) \lesssim l^{d-1},
$$

as desired.

Proof of Lemma 1. To prove (1i) notice that a minimizer $(u, \mathbf{b})$ in $\mathcal{A}^{\text {per }}\left(Q_{L}\right)$, when thought of as peridically extended and then restricted to $Q_{L}$, is also a candidate in $\mathcal{A}\left(Q_{L}\right)$, and that $E^{\text {per }}\left(u, \mathbf{b}, Q_{L}\right) \geq E\left(u, \mathbf{b}, Q_{L}\right)$, leading to $\sigma^{\text {per }}\left(Q_{L}\right) \geq \sigma\left(Q_{L}\right)$. Similarly, we have $\sigma^{0}\left(Q_{L}\right) \geq \sigma\left(Q_{L}\right)$.

To prove (iii), let $k$ be a positive integer and consider a minimizer $(u, \mathbf{b})$ in $\mathcal{A}\left(Q_{k L}\right)$. We decompose $Q_{k L}$ in $k^{d-1}$ cubes $\left\{Q_{L}^{i}\right\}$ of side length $L$ touching the lower boundary, and a large box above. Since by definition of $\sigma, E\left(u, \mathbf{b}, Q_{L}^{i}\right) \geq L^{d-1} \sigma\left(Q_{L}\right)$, we obtain

$$
\sigma\left(Q_{k L}\right)=\frac{E\left(u, \mathbf{b}, Q_{k L}\right)}{(k L)^{d-1}} \geq \frac{1}{k^{d-1}} \sum_{i=1}^{k^{d-1}} \frac{E\left(u, \mathbf{b}, Q_{L}^{i}\right)}{L^{d-1}} \geq \sigma\left(Q_{L}\right) .
$$

Statement (iii) comes from concatenating $k^{d-1}$ translated copies of a minimizer in $\mathcal{A}^{0}\left(Q_{L}\right)$ and finally extending $\mathbf{b}$ by 0 on $x_{d} \geq L$. There is no added interface if each copy is the even reflection of its neighbour across the cubic face they share. We refer to 
Sections 3.6 to 3.8 in [3] for the continuity and trace properties that make it possible to cut and paste BV functions in neighbourhing cubes.

To prove (iv) notice that by (iii) it suffices to show that $\sigma^{0}\left(Q_{L}\right) \lesssim 1$ for $L \in[1,2]$. This is seen by constructing $(u, \mathbf{b}) \in \mathcal{A}\left(Q_{L}\right)$. Indeed, let $u\left(x_{1}, \ldots, x_{d-1}\right):=\operatorname{sign} x_{1}$ and

$$
\mathbf{b}\left(x_{1}, \ldots, x_{d-1}, x_{d}\right)= \begin{cases}\frac{x_{1}}{\sqrt{x_{1}^{2}+x_{d}^{2}}} \mathbf{e}_{d}-\frac{x_{d}}{\sqrt{x_{1}^{2}+x_{d}^{2}}} \mathbf{e}_{1}, & \text { if } x_{1}^{2}+x_{d}^{2} \leq L^{2}, \\ 0 & \text { otherwise }\end{cases}
$$

We notice that inside $\left\{x_{1}^{2}+x_{d}^{2} \leq L^{2}\right\}$, this two-dimensional vector field is the rotated gradient of the "stream function" $\psi\left(x_{1}, x_{d}\right)=\sqrt{x_{1}^{2}+x_{d}^{2}}-L$, and thus divergence-free and tangential to $\left\{x_{1}^{2}+x_{d}^{2}=L^{2}\right\}$. Hence its trivial extension is weakly divergence-free. It vanishes on $\Gamma_{L}$, and satisfies $\mathbf{b} \cdot \mathbf{e}_{d}=\operatorname{sign} x_{1}=u$ on $\underline{Q_{L}}$, so that the boundary conditions are also satisfied.

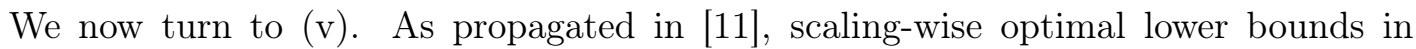
pattern-forming variational problems typically rely on interpolation inequalities that capture the leading-order competition between the energy contributions. Here, the interpolation estimate involves the $B V$ norm and the $\dot{H}^{-\frac{1}{2}}$ norm - we now give an elementary proof. Choosing $l$ to be sufficiently large but of order one, it is enough to show for $(u, \mathbf{b}) \in \mathcal{A}\left(Q_{L}\right)$ that

$$
L^{d-1} \lesssim l E\left(u, \mathbf{b}, Q_{L}\right)+\frac{L^{d-1}}{l^{2}} .
$$

By Young's inequality, for this it suffices to establish for $l \ll L$

$$
\left|\underline{Q_{L-2 l}}\right| \lesssim l \int_{\underline{Q_{L}}}|\nabla u|+\left(\frac{L^{d-1}}{l} \int_{Q_{L}}|\mathbf{b}|^{2}\right)^{\frac{1}{2}} .
$$

To this purpose, we fix a cut-off function $\eta \in[0,1]$ with

$$
\eta=\left\{\begin{array}{ccc}
1 & \text { on } \quad\left(-\frac{L-2 l}{2}, \frac{L-2 l}{2}\right)^{d-1} \times\{0\}, \\
0 & \text { out of } \quad\left(-\frac{L-l}{2}, \frac{L-l}{2}\right)^{d-1} \times[0, l),
\end{array}\right\} \quad \text { while } \quad|\nabla \eta| \lesssim \frac{1}{l} .
$$

By convolution at scale $l$, we construct $\tilde{u} \in[-1,1]$ such that

$$
\int_{\underline{Q_{L-l}}}|\tilde{u}-u| \lesssim l \int_{\underline{Q_{L}}}|\nabla u| \quad \text { while } \quad|\nabla \tilde{u}| \lesssim \frac{1}{l}
$$

In particular, we obtain from this and the support condition in (21)

$$
\int_{\mathbb{R}^{d-1}}|\eta u(u-\tilde{u})| \lesssim l \int_{\underline{Q_{L}}}|\nabla u| .
$$

We now test (2) with $\zeta=\eta \tilde{u}$, where we think of $\tilde{u}$ as being extended in a constant way to $x_{d}>0$. This yields

$$
\int_{\mathbb{R}^{d-1}} \eta \tilde{u} u=-\int_{\mathbb{R}^{d}} \nabla \zeta \cdot \mathbf{b},
$$


which in view of $|\nabla \zeta| \lesssim 1 / l$ (see the last items in (21) and (22) ) and the Hölder inequality, implies

$$
\int_{\mathbb{R}^{d-1}} \eta \tilde{u} u \lesssim\left(\frac{L^{d-1}}{l} \int_{Q_{L}}|\mathbf{b}|^{2}\right)^{\frac{1}{2}}
$$

Because of the non-convex constraint in form of $u^{2}=1$, the sum of (23) and (24) yields

$$
\int_{\mathbb{R}^{d-1}} \eta \lesssim l \int_{\underline{Q_{L}}}|\nabla u|+\left(\frac{L^{d-1}}{l} \int_{Q_{L}}|\mathbf{b}|^{2}\right)^{\frac{1}{2}}
$$

which by (21) turns into (20).

To prove (vi), we let $(u, \mathbf{b})$ be an optimal configuration in $\mathcal{A}\left(Q_{L}\right)$. On one of the $2^{d-1}$ (horizontal) quadrants of $Q_{L}$, the energy per area does not exceed the original one, w.l.o.g. we may assume that this is the case for $(0, L / 2)^{d-1} \times(0, L)$. We extend the restriction of $(u, \mathbf{b})$ to this quadrant by reflections to obtain a candidate $(\bar{u}, \overline{\mathbf{b}}) \in \mathcal{A}^{\text {per }}\left(Q_{L}\right)$. We do this iteratively in the horizontal directions $i=1, \ldots, d-1: \mathbf{b} \cdot \mathbf{e}_{i}$ is reflected evenly across $x_{i}=0$ in order to avoid a jump, $u$ and all the other components of $\mathbf{b}$ are reflected oddly. This way, we obtain a new configuration $(\bar{u}, \overline{\mathbf{b}})$ on $Q_{L}$, the energy of which exceeds the one of $(u, \mathbf{b})$ by at most the additional interfacial energy $2(d-1) L^{d-2}$. (We refer again to Sections 3.6 to 3.8 in [3] for the results that allow us to cut and paste BV functions.) Extending $(\bar{u}, \overline{\mathbf{b}})$ periodically in the horizontal directions adds interfacial energy by at most the same amount.

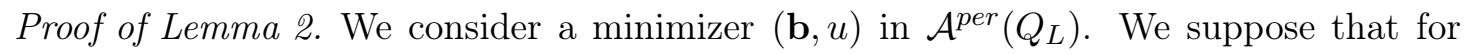
some $\delta \in(0,+\infty)$ to be chosen later, there holds $F(\beta, l) \leq \delta$, for some $l$ and $\beta$ with $L \geq l \gg 1$ and $\beta \in[-1 / 2,1 / 2]$. We choose a smaller scale $\rho$ such that

$$
\rho:=\operatorname{argmin}\left\{\int_{\Gamma_{r}}\left|\mathbf{b}-\beta \mathbf{e}_{d}\right|^{2} \mid r \in\left[\frac{3}{4} l, l\right]\right\} .
$$

By Fubini's theorem, this choice of $\rho$ implies that

$$
\frac{1}{\rho^{d-1}} \int_{\Gamma_{\rho}}\left|\mathbf{b}-\beta \mathbf{e}_{d}\right|^{2} \lesssim \frac{1}{l^{d}} \int_{Q_{l}}\left|\mathbf{b}-\beta \mathbf{e}_{d}\right|^{2} \stackrel{\square[}{=} F(\beta, l) .
$$

Let $v_{0}$ be the solution of the over-relaxed problem (6) in $Q_{\rho}$ with flux boundary data $\left(\mathbf{b}-\beta \mathbf{e}_{d}\right) \cdot \nu$ on $\Gamma_{\rho}$. We note that the Dirichlet energy of $-\nabla v_{0}$ is less than the squared $\mathrm{L}^{2}$-norm of $\mathbf{b}-\beta \mathbf{e}_{d}$, as $-\nabla v_{0}$ is obtained by relaxation. Extend $v_{0}$ harmonically to negative values of $x_{d}$ by odd reflection (this is possible because $v_{0}=0$ on $\underline{Q_{\rho}}$ ). We now shift the problem by $(\tilde{\beta}-\beta) \mathbf{e}_{d}$, where

$$
\tilde{\beta}:=\beta+\partial_{d} v_{0}(0) .
$$

By the fact that $\nabla v_{0}(0)=\partial_{d} v_{0}(0) \mathbf{e}_{d}$ and sub-harmonicity of $\left|\nabla v_{0}\right|^{2}$, we have

$$
(\tilde{\beta}-\beta)^{2} \stackrel{(26]}{=}\left|\nabla v_{0}(0)\right|^{2} \lesssim \frac{1}{\rho^{d}} \int_{Q_{\rho}}\left|\nabla v_{0}\right|^{2} \leq \frac{1}{\rho^{d}} \int_{Q_{\rho}}\left|\mathbf{b}-\beta \mathbf{e}_{d}\right|^{2} \stackrel{(7)}{\lesssim} F(\beta, l) .
$$


For $\theta \in(0,1 / 2]$ to be chosen later, by the triangle inequality we obtain

$$
\begin{aligned}
\int_{\underline{Q_{\theta l}}}|\nabla u|+\int_{Q_{\theta l}} & \frac{1}{2}\left|\mathbf{b}-\tilde{\beta} \mathbf{e}_{d}\right|^{2} \\
& \leq \int_{\underline{Q_{\theta l}}}|\nabla u|+\int_{Q_{\theta l}}\left|\mathbf{b}-\beta \mathbf{e}_{d}+\nabla v_{0}\right|^{2}+\int_{Q_{\theta l}}\left|\nabla v_{0}-(\tilde{\beta}-\beta) \mathbf{e}_{d}\right|^{2} .
\end{aligned}
$$

We will treat the sum of the two first terms and the third term separately. For the third term, the mean value property (Theorem 2.1 in [15]) yields

$$
\frac{1}{(\theta l)^{d}} \int_{Q_{\theta l}}\left|\nabla v_{0}-(\tilde{\beta}-\beta) \mathbf{e}_{d}\right|^{2} \stackrel{(26)}{=} \frac{1}{(\theta l)^{d}} \int_{Q_{\theta l}}\left|\nabla v_{0}-\nabla v_{0}(0)\right|^{2} \lesssim(\theta l)^{2} \sup _{Q_{\theta l}}\left|\nabla^{2} v_{0}\right|^{2} .
$$

By inner regularity of harmonic functions (for instance Theorem 2.10 in [15], followed by the mean value property), recalling that $\theta \in(0,1 / 2]$ we thus get

$$
\frac{1}{(\theta l)^{d}} \int_{Q_{\theta l}}\left|\nabla v_{0}-(\tilde{\beta}-\beta) \mathbf{e}_{d}\right|^{2} \lesssim(\theta l)^{2} \frac{1}{\rho^{d+2}} \int_{Q_{\rho}}\left|\nabla v_{0}\right|^{2} \stackrel{(27)}{\lesssim} \theta^{2} F(\beta, l) .
$$

We now compare the first two terms on the right hand side of inequality (28) with the energy of the relaxed problem, using Lemma 5. Define $\overline{\mathbf{b}}$ as the solution to the relaxed problem (10) on $Q_{\rho}$ with flux boundary values $\mathbf{b} \cdot \nu$ across $\Gamma_{\rho}$. By Lemma 5 , one can find a candidate $(\tilde{u}, \tilde{\mathbf{b}})$ in $\mathcal{A}^{\mathbf{b} \cdot \nu}\left(Q_{\rho}\right)$ such that

$$
\int_{\underline{Q_{\rho}}}|\nabla \tilde{u}|+\int_{Q_{\rho}} \frac{1}{2}|\tilde{\mathbf{b}}-\overline{\mathbf{b}}|^{2} \lesssim \rho^{d-1}
$$

Thus, by the triangle inequality, we have

$$
\int_{\underline{Q_{\rho}}}|\nabla \tilde{u}|+\int_{Q_{\rho}}\left|\tilde{\mathbf{b}}-\beta \mathbf{e}_{d}+\nabla v_{0}\right|^{2} \lesssim \int_{Q_{\rho}}\left|\overline{\mathbf{b}}-\beta \mathbf{e}_{d}+\nabla v_{0}\right|^{2}+\rho^{d-1} .
$$

Furthermore, we note that $(u, \mathbf{b})$ is almost a minimizer in $\mathcal{A}^{\mathbf{b} \cdot \nu}\left(Q_{\rho}\right)$ (up to the interfacial energy concentrated in $\partial\left(\underline{Q_{\rho}}\right)$ ), so that we have

$$
E\left(u, \mathbf{b}, Q_{\rho}\right) \leq E\left(\tilde{u}, \tilde{\mathbf{b}}, Q_{\rho}\right)+C \rho^{d-2} .
$$

Applying Lemma 7 to substract $-\nabla v_{0}+\beta \mathbf{e}_{d}$ from the fields, this turns into

$$
\int_{\underline{Q_{\rho}}}|\nabla u|+\int_{Q_{\rho}} \frac{1}{2}\left|\mathbf{b}-\beta \mathbf{e}_{d}+\nabla v_{0}\right|^{2} \leq \int_{\underline{Q_{\rho}}}|\nabla \tilde{u}|+\int_{Q_{\rho}} \frac{1}{2}\left|\tilde{\mathbf{b}}-\beta \mathbf{e}_{d}+\nabla v_{0}\right|^{2}+C \rho^{d-2} .
$$

Combining the last estimate with (30) yields

$$
\int_{\underline{Q_{\rho}}}|\nabla u|+\int_{Q_{\rho}}\left|\mathbf{b}-\beta \mathbf{e}_{d}+\nabla v_{0}\right|^{2} \lesssim \int_{Q_{\rho}}\left|\overline{\mathbf{b}}-\beta \mathbf{e}_{d}+\nabla v_{0}\right|^{2}+\rho^{d-1} .
$$

We now crucially use Lemma 4 to estimate the minimum energy of the relaxed problem in $Q_{\rho}$. By the choice of $\rho$, we have

$$
\int_{\Gamma_{\rho}}\left(\left(\mathbf{b}-\beta \mathbf{e}_{d}\right) \cdot \nu\right)^{2} \stackrel{(25)}{\lesssim} l^{d-1} F(\beta, l) \leq \delta l^{d-1} .
$$


Thus for sufficiently small $\delta$, by Lemma 4 ,

$$
\int_{Q_{\rho}}\left|\overline{\mathbf{b}}-\beta \mathbf{e}_{d}+\nabla v_{0}\right|^{2} \lesssim\left(\int_{\Gamma_{\rho}}\left(\left(\overline{\mathbf{b}}-\beta \mathbf{e}_{d}\right) \cdot \nu\right)^{2}\right)^{\frac{d}{d-1}} \stackrel{\frac{(25)}{\lesssim}}{\lesssim} l^{d} F(\beta, l)^{\frac{d}{d-1}},
$$

so that, restricting to the cube $Q_{\theta l} \subset Q_{\rho}$, we get

$$
\begin{aligned}
\int_{\underline{Q_{\theta l}}}|\nabla u|+\int_{Q_{\theta l}} \mid \mathbf{b}-\beta & \beta \mathbf{e}_{d}+\left.\nabla v_{0}\right|^{2} \leq \int_{\underline{Q_{\rho}}}|\nabla u|+\int_{Q_{\rho}}\left|\mathbf{b}-\beta \mathbf{e}_{d}+\nabla v_{0}\right|^{2} \\
& \stackrel{(31)}{\lesssim} \int_{Q_{\rho}}\left|\overline{\mathbf{b}}-\beta \mathbf{e}_{d}+\nabla v_{0}\right|^{2}+l^{d-1} \lesssim l^{d} F(\beta, l)^{\frac{d}{d-1}}+l^{d-1} .
\end{aligned}
$$

We may now conclude: inserting the estimates (29) and (32) into (28), we obtain

$$
F(\tilde{\beta}, \theta l) \leq C\left(\theta^{2} F(\beta, l)+\frac{1}{\theta^{d}} F(\beta, l)^{\frac{d}{d-1}}+\frac{1}{\theta^{d} l}\right) .
$$

We first choose $\theta \ll 1$ such that

$$
C \theta^{2} F(\beta, l) \leq \frac{1}{2} \theta F(\beta, l)
$$

and then $\delta \ll 1$ such that

$$
\frac{C}{\theta^{d}} F(\beta, l)^{\frac{d}{d-1}} \leq \frac{1}{2} \theta F(\beta, l),
$$

which, combined with (27) yields (9).

Proof of Lemma 3. Let $\delta \in(0,+\infty)$ be arbitrary, to be chosen later. Consider a minimizer $(u, \mathbf{b})$ in $\mathcal{A}^{\text {per }}\left(Q_{L}\right)$. Fix $\theta \in(0,1 / 2]$ according to Lemma 2 . Without loss of generality, we ask that $l=\theta^{N} L$ for some positive integer $N$. By induction over $n$, we will prove that there exists a sequence of shifts $\left\{\beta_{n}\right\}_{n=1}^{N}$ in $[-1 / 2,1 / 2]$, such that for $n=1, \ldots, N$, there holds

$$
F\left(\beta_{n}, \theta^{n} L\right) \leq \theta^{n} F(0, L)+\frac{C \theta^{n+1}}{L} \sum_{m=1}^{n} \theta^{-2 m} \text { and }\left|\beta_{n}\right| \leq C\left(F(0, L)^{1 / 2}+\frac{1}{\sqrt{\theta^{n} L}}\right),
$$

with $C \in(0,+\infty)$ to be chosen later. In particular we remark that for $n \leq N$, the first part of (33) implies

$$
\begin{aligned}
F\left(\beta_{n}, \theta^{n} L\right) & \leq \theta^{n} F(0, L)+\frac{C \theta^{n+1}}{L} \frac{\theta^{-2(n+1)}-1}{\theta^{-2}-1} \\
& =\theta^{n} F(0, L)+\frac{C \theta^{n+1}}{L} \frac{\theta^{-2 n}-\theta^{2}}{1-\theta^{2}} \\
& \leq \theta^{n} F(0, L)+\frac{C}{1-\theta^{2}} \frac{1}{\theta^{n-1} L} .
\end{aligned}
$$

Letting $\beta_{0}:=0$, the inequalities in (33) hold trivially for $n=0$. We now pass from $n$ to $n+1$. Suppose that (33) and (34) hold at all steps from 0 to $n$ with $0 \leq n \leq N-1$. We note that (33) and (34) imply that the assumption (8) from Lemma 2 is satisfied 
provided $\delta$ in the present proof is chosen sufficiently small. Denoting by $C_{0}$ the implicit constant in (9), there exists $\beta_{n+1}$ such that

$F\left(\beta_{n+1}, \theta^{n+1} L\right) \leq \theta F\left(\beta_{n}, \theta^{n} L\right)+\frac{C_{0}}{\theta^{n} L} \stackrel{\text { (33) }}{\leq} \stackrel{\text { at step } n}{\leq} \theta^{n+1} F(0, L)+\frac{C \theta^{n+1}}{L} \sum_{m=1}^{n} \theta^{-2 m}+\frac{C_{0}}{\theta^{n} L}$.

This is consistent with (33) provided we choose $C$ such that $C \geq C_{0}$. Furthermore, by (9), we have

$$
\left|\beta_{n+1}-\beta_{n}\right| \leq C_{0} F\left(\beta_{n}, \theta^{n} L\right)^{\frac{1}{2}}
$$

We thus control $\beta_{n+1}$ by

$$
\begin{array}{rll}
\left|\beta_{n+1}\right| & \leq & \sum_{m=0}^{n}\left|\beta_{m+1}-\beta_{m}\right| \\
& \leq & C_{0} \sum_{m=0}^{n} F\left(\beta_{m}, \theta^{m} L\right)^{\frac{1}{2}} \\
& \leq \\
& C_{0}\left(\sum_{m=0}^{n}\left(\theta^{m} F(0, L)\right)^{\frac{1}{2}}+\sqrt{\frac{C}{1-\theta^{2}}} \sum_{m=1}^{n} \frac{1}{\sqrt{\theta^{m-1} L}}\right) \\
\leq & C_{0} \frac{1}{1-\sqrt{\theta}} F(0, L)^{\frac{1}{2}}+C_{0} \sqrt{\frac{C}{1-\theta^{2}}} \frac{1}{\sqrt{\theta}-1} \frac{1}{\sqrt{\theta^{n} L}}
\end{array}
$$

This is consistent with (33) at step $n+1$ and implies in particular $\beta_{n+1} \in[-1 / 2,1 / 2]$ provided we choose $C$ such that

$$
C \geq \max \left\{C_{0} \frac{1}{1-\sqrt{\theta}}, C_{0} \sqrt{\frac{C}{1-\theta^{2}}} \frac{1}{\sqrt{\theta}^{-1}-1}\right\} .
$$

Using (34) for $n=N$, we obtain

$$
F\left(\beta_{N}, l\right) \lesssim F(0, L)+\frac{1}{l}
$$

By the triangle inequality, using the fact that $F$ is a volume average, we may remove the shift:

$$
F(0, l) \lesssim F\left(\beta_{N}, l\right)+\beta_{N}^{2} \stackrel{\sqrt[366]{,(33)}}{\lesssim} F(0, L)+\frac{1}{l}
$$

Proof of Lemma 4. We first note that $\mathbf{b}$ being a minimizer of the relaxed problem (10) implies that for $\beta \in \mathbb{R}, \mathbf{b}-\beta \mathbf{e}_{d}$ is a minimizer of the shifted relaxed problem:

$$
\min \left\{\begin{array}{l|l}
\int_{Q_{L}} \frac{1}{2}|\tilde{\mathbf{b}}|^{2} \mid \begin{array}{cccc}
\nabla \cdot \tilde{\mathbf{b}} & = & 0 & \text { in } Q_{L}, \\
\tilde{\mathbf{b}} \cdot \nu & \in & -1+\beta, 1+\beta] & \text { on } \frac{Q_{L}}{\tilde{\mathbf{b}} \cdot \nu}, \\
\Gamma_{L}
\end{array}
\end{array}\right\}
$$


Indeed, if $\tilde{\mathbf{b}}$ is a candidate for the problem (37), writing $\mathbf{e}_{d}=\nabla x_{d}$, we obtain by integration by parts that $\int_{Q_{L}}\left(\mathbf{b}-\beta \mathbf{e}_{d}-\tilde{\mathbf{b}}\right) \cdot \beta \mathbf{e}_{d}=0$ so that

to the effect of

$$
\int_{Q_{L}} \mathbf{b} \cdot\left(\beta \mathbf{e}_{d}\right)-\frac{1}{2}\left|\beta \mathbf{e}_{d}\right|^{2}=\int_{Q_{L}} \tilde{\mathbf{b}} \cdot\left(\beta \mathbf{e}_{d}\right)+\frac{1}{2}\left|\beta \mathbf{e}_{d}\right|^{2}
$$

$$
\int_{Q_{L}} \frac{1}{2}|\tilde{\mathbf{b}}|^{2}-\int_{Q_{L}} \frac{1}{2}\left|\mathbf{b}-\beta \mathbf{e}_{d}\right|^{2}=\int_{Q_{L}} \frac{1}{2}\left|\tilde{\mathbf{b}}+\beta \mathbf{e}_{d}\right|^{2}-\int_{Q_{L}} \frac{1}{2}|\mathbf{b}|^{2} .
$$

The last term is non-negative as $\tilde{\mathbf{b}}+\beta \mathbf{e}_{d}$ is a candidate for the relaxed problem (10), of which $\mathbf{b}$ is a minimizer. Thus, recalling that $\beta \in[-1 / 2,1 / 2]$ and considering a minimizer $\tilde{\mathbf{b}}$ of the more constrained problem

$$
\min \left\{\int_{Q_{L}} \frac{1}{2}|\tilde{\mathbf{b}}|^{2} \mid \begin{array}{cccc}
\nabla \cdot \mathbf{b} & = & 0 & \text { in } Q_{L}, \\
\tilde{\mathbf{b}} \cdot \nu & \in & {[-1 / 2,-1 / 2]} & \text { on } \frac{Q_{L}}{\Gamma_{L}}, \\
\tilde{\mathbf{b}} \cdot \nu & = & \left(\mathbf{b}-\beta \mathbf{e}_{d}\right) \cdot \nu & \text { on }
\end{array}\right\}
$$

there holds

$$
\int_{Q_{L}}\left|\mathbf{b}-\beta \mathbf{e}_{d}\right|^{2} \leq \int_{Q_{L}}|\tilde{\mathbf{b}}|^{2}
$$

Consider also the solution $-\nabla v_{0}$ to the over-relaxed problem (6) in $Q_{L}$ with flux boundary data given by $\tilde{\mathbf{b}} \cdot \nu$ on $\Gamma_{L}$. For $\tilde{\mathbf{b}}$ and $-\nabla v_{0}$ it is clear that Lemma 7, but also version of Lemma 6 hold (replacing the constraint $\mathbf{b} \cdot \nu \in[-1,1]$ by $\mathbf{b} \cdot \nu \in[-1 / 2,1 / 2]$ only affects the implicit constant), so that

$$
\int_{Q_{L}}|\tilde{\mathbf{b}}|^{2}-\int_{Q_{L}}\left|\nabla v_{0}\right|^{2}=\int_{Q_{L}}\left|\tilde{\mathbf{b}}+\nabla v_{0}\right|^{2} \lesssim\left(\int_{\Gamma_{L}}\left(\left(\mathbf{b}-\beta \mathbf{e}_{d}\right) \cdot \nu\right)^{2}\right)^{\frac{d}{d-1}} .
$$

Hence, using (38) and once more Lemma 7 , we get as desired

$$
\int_{Q_{l}}\left|\mathbf{b}-\beta \mathbf{e}_{d}+\nabla v_{0}\right|^{2} \lesssim\left(\int_{\Gamma_{l}}\left(\left(\mathbf{b}-\beta \mathbf{e}_{d}\right) \cdot \nu\right)^{2}\right)^{\frac{d}{d-1}} .
$$

Proof of Lemma 5, Let $\overline{\mathbf{b}}$ be a minimizer for the relaxed problem (10) in $Q_{L}$ with flux boundary condition $g$. Divide $Q_{L}$ into $\mathcal{O}\left(L^{d-1}\right)$ small cubes $Q_{i}$ with side length $l \in[1,2]$ sitting on the bottom and a large box above of height $L-l$ and sides of length $L$. Divide each $\underline{Q_{i}}$ into two boxes $\underline{Q_{i}^{+}}$and $\underline{Q_{i}^{-}}$, such that $\operatorname{area}\left({\underline{Q_{i}}}^{+}\right)-\operatorname{area}\left(\underline{Q_{i}}{ }^{-}\right)=\int_{\underline{Q_{i}}} \overline{\mathbf{b}} \cdot \nu \in$ $\left[-l^{d-1}, l^{d-1}\right]$. Then define $u$ on $\underline{Q_{i}}$ so that

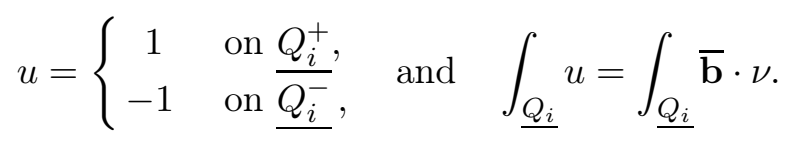

The interfacial energy in $Q_{i}$ is given by $\int_{\underline{Q_{i}}}|\nabla u|=2 l^{d-2}$ (or zero if $\int_{\underline{Q_{i}}} \overline{\mathbf{b}} \cdot \nu= \pm l^{d-1}$ ). Taking into account the interface between the cubes $\underline{Q_{i}}$, the global interfacial energy in 
$\underline{Q_{L}}$ thus satisfies

$$
\int_{\underline{Q_{L}}}|\nabla u| \leq\left(\frac{L}{l}\right)^{d-1} 2 l^{d-2}+2 d \frac{L}{l} \quad 2 L^{d-2} \lesssim L^{d-1}
$$

It remains to modify the field $\overline{\mathbf{b}}$ so that it becomes compatible with $u$. In each $Q_{i}$, we solve the following Neumann problem (which is solvable by (39))

$$
\left\{\begin{array}{llll}
-\Delta v_{i} & =0 & & \text { in } Q_{i} \\
-\nabla v_{i} \cdot \nu & =0 & & \text { on } \partial Q_{i} \backslash \underline{Q_{i}} \\
-\nabla v_{i} \cdot \nu & =u-\overline{\mathbf{b}} \cdot \nu & & \text { on } \underline{Q_{i}} .
\end{array}\right.
$$

As $u= \pm 1$ and $|\overline{\mathbf{b}} \cdot \nu| \leq 1$ on $\underline{Q_{i}}$, we may apply Lemma 11 and there holds $\int_{Q_{i}}\left|\nabla v_{i}\right|^{2} \lesssim 1$. Define a field $\mathbf{b}$ by

$$
\mathbf{b}:= \begin{cases}\overline{\mathbf{b}}-\nabla v_{i} & \text { on } Q_{i} \\ \overline{\mathbf{b}} & \text { in } Q_{L} \backslash \bigcup_{i} Q_{i}\end{cases}
$$

By construction, $(u, \mathbf{b}) \in \mathcal{A}^{g}\left(Q_{L}\right)$ and there holds

$$
\int_{Q_{L}}|\mathbf{b}-\overline{\mathbf{b}}|^{2}=\sum_{i} \int_{Q_{i}}\left|\nabla v_{i}\right|^{2} \lesssim L^{d-1}
$$

Proof of Lemma 6. Clearly, b and $v_{0}$ satisfy the assumptions of Lemma 7, so we have

$$
\int_{Q_{L}} \frac{1}{2}\left|\mathbf{b}+\nabla v_{0}\right|^{2}=\int_{Q_{L}} \frac{1}{2}|\mathbf{b}|^{2}-\int_{Q_{L}} \frac{1}{2}\left|\nabla v_{0}\right|^{2}=E_{r e l}^{g}\left(Q_{L}\right)-E_{0}^{g}\left(Q_{L}\right) .
$$

Let $v$ be the minimizer of the dual to the relaxed problem on $Q_{L}$, see (111), and let $w:=v-v_{0}$. There holds

$$
\begin{aligned}
E_{r e l}^{g}\left(Q_{L}\right)-E_{0}^{g}\left(Q_{L}\right) & =-\int_{Q_{L}} \frac{1}{2}|\nabla v|^{2}-\int_{\underline{Q_{L}}}|v|+\int_{\Gamma_{L}} v g-\int_{Q_{L}} \frac{1}{2}\left|\nabla v_{0}\right|^{2} \\
& =-\int_{Q_{L}} \frac{1}{2}\left|\nabla v-\nabla v_{0}\right|^{2}-\int_{Q_{L}} \nabla v \cdot \nabla v_{0}-\int_{\underline{Q_{L}}}|v|+\int_{\Gamma_{L}} v g .
\end{aligned}
$$

We now integrate by parts the mixed term and appealing to ([6), we get

$$
E_{\text {rel }}^{g}\left(Q_{L}\right)-E_{0}^{g}\left(Q_{L}\right)=-\int_{Q_{L}} \frac{1}{2}|\nabla w|^{2}+\int_{\underline{Q_{L}}}\left(-|w|+w \partial_{d} v_{0}\right) .
$$


Next, denoting $V:=\left(\int_{\underline{Q_{L}}}\left(\partial_{d} v_{0}\right)^{2}\right)^{1 / 2}$, by Hölder's inequality, Lemma 9 for $\epsilon \in(0,1]$, and Young's inequality, we obtain

$$
\begin{aligned}
& E_{r e l}^{g}\left(Q_{L}\right)-E_{0}^{g}\left(Q_{L}\right) \leq-\int_{Q_{L}} \frac{1}{2}|\nabla w|^{2}-\int_{\underline{Q_{L}}}|w|+\left(\int_{\underline{Q_{L}}} w^{2}\right)^{\frac{1}{2}} V \\
& \leq-\int_{Q_{L}} \frac{1}{2}|\nabla w|^{2}+C(\epsilon L)^{\frac{1}{2}}\left(\int_{Q_{L}}|\nabla w|^{2}\right)^{\frac{1}{2}} V+\left(\frac{C V}{(\epsilon L)^{\frac{d-1}{2}}}-1\right) \int_{\underline{Q_{L}}}|w| \\
& \leq \frac{1}{2} C^{2} \epsilon L V^{2}+\left(\frac{C V}{(\epsilon L)^{\frac{d-1}{2}}}-1\right) \int_{\underline{Q_{L}}}|w| .
\end{aligned}
$$

From Lemma 10 applied to $v_{0}$ and the assumption $\int_{\Gamma_{L}} g^{2} \ll L^{d-1}$, which we rewrite as $\int_{\Gamma_{L}} g^{2} \leq c L^{d-1}$ for some $c \in(0,+\infty)$, we know that

$$
V=\left(\int_{\underline{Q_{L}}}\left(\partial_{d} v_{0}\right)^{2}\right)^{\frac{1}{2}} \lesssim\left(\int_{\Gamma_{L}} g^{2}\right)^{\frac{1}{2}} \lesssim c^{\frac{1}{2}} L^{\frac{d-1}{2}}
$$

Hence, if $c$ is small enough, depending only on $d$, we may choose $\epsilon \in(0,1]$ such that $(\epsilon L)^{(d-1) / 2} \geq C V$, so that the second term on the right hand side of (40) is non-positive. Using (41) in (40), we thus obtain as desired

$$
E_{r e l}^{g}\left(Q_{L}\right)-E_{0}^{g}\left(Q_{L}\right) \lesssim V^{\frac{2}{d-1}+2} \lesssim\left(\int_{\Gamma_{l}} g^{2}\right)^{\frac{d}{d-1}} .
$$

Proof of Lemma [, Using the condition $\nabla \cdot \mathbf{b}=0$ to integrate by parts, we get

$$
\int_{Q_{L}} \mathbf{b} \cdot \nabla v_{0}=-\int_{\Gamma_{L}} v_{0} \mathbf{b} \cdot \nu=-\int_{\Gamma_{L}} v_{0} g=\int_{\Gamma_{L}} v_{0} \nabla v_{0} \cdot \nu=-\int_{Q_{L}}\left|\nabla v_{0}\right|^{2} .
$$

Hence

$$
\int_{Q_{L}} \frac{1}{2}\left|\mathbf{b}+\nabla v_{0}\right|^{2}=\int_{Q_{L}} \frac{1}{2}|\mathbf{b}|^{2}+\int_{Q_{L}} \mathbf{b} \cdot \nabla v_{0}+\int_{Q_{L}} \frac{1}{2}\left|\nabla v_{0}\right|^{2}=\int_{Q_{L}} \frac{1}{2}|\mathbf{b}|^{2}-\int_{Q_{L}} \frac{1}{2}\left|\nabla v_{0}\right|^{2} .
$$

Proof of Lemma 8, This proof is similar to that of Lemma 3.3 in 2]. The idea is to replace the constraints by a linear (thus concave) maximization problem. When the constraints for $\mathbf{b}$ are not met, the supremum is infinite and such candidates cannot be minimizers. The condition $\left|\int_{\Gamma_{L}} g\right| \leq L^{d-1}$ ensures that the class on which we minimize 
for the relaxed problem is not empty. This minimization problem can then be stated as

$$
\begin{aligned}
& E_{r e l}^{g}\left(Q_{L}\right)=\inf _{\mathbf{b}}\left\{\int_{Q_{L}} \frac{1}{2}|\mathbf{b}|^{2} \mid \begin{array}{cccc}
\nabla \cdot \mathbf{b} & = & 0 & \text { in } Q_{L}, \\
\mathbf{b} \cdot \nu & \in & {[-1,1]} & \text { on } \frac{Q_{L}}{\mathbf{b}_{L}}, \nu \\
\Gamma_{L} & g & \text { on }
\end{array}\right\} \\
& =\inf _{\mathbf{b}, u}\left\{\int_{Q_{L}} \frac{1}{2}|\mathbf{b}|^{2} \mid \begin{array}{cccccc}
\mathbf{b} & \in & \mathrm{L}^{2}\left(Q_{L}, \mathbb{R}^{d}\right), & \nabla \cdot \mathbf{b}=0 & \text { in } Q_{L}, \\
u & \in & \mathrm{L}^{2}\left(\underline{Q_{L}},[-1,1]\right), & \mathbf{b} \cdot \nu=u & \text { on } \underline{Q_{L}}, \\
\mathbf{b} \cdot \nu & =g & \text { on } \frac{\Gamma_{L}}{\Gamma_{L}}
\end{array}\right\} \\
& =\inf _{\mathbf{b}, u} \sup _{v}\left\{\int_{Q_{L}}\left(\frac{1}{2}|\mathbf{b}|^{2}+\mathbf{b} \cdot \nabla v\right)+\int_{\underline{Q_{L}}} v u+\int_{\Gamma_{L}} v g \mid \begin{array}{ccc}
\mathbf{b} & \in & \mathrm{L}^{2}\left(Q_{L}, \mathbb{R}^{d}\right), \\
u & \in & \mathrm{L}^{2}\left(Q_{L},[-1,1]\right), \\
v & \in & \frac{Q^{1}}{\mathrm{H}^{1}}\left(Q_{L}\right) .
\end{array}\right\}
\end{aligned}
$$

The infimum being taken for a convex functional, and the supremum for an affine one, we use a classical min - max theorem (see for instance [7, Chapter I, Proposition 1.1) to change the order of the operations. To see that we may apply this proposition, let us denote the functional in the last line by $K((u, \mathbf{b}), v)$; it is defined on the product of the spaces $E:=\mathrm{L}^{2}\left(\underline{Q_{L}}\right) \times \mathrm{L}^{2}\left(Q_{L}, \mathbb{R}^{d}\right)$ and $\mathrm{H}^{1}\left(Q_{L}\right)$, equiped with the weak topology. Consider the convex subset $A:=E \cap\{(u, \mathbf{b}), u \in[-1,1]\}$. The functional $K$ is linear and bounded in the variable $v$, thus concave and weakly continuous. It is also convex and weakly lower semi continuous in the variable $(u, \mathbf{b})$ on $A$. If $\widehat{\mathbf{b}}=-\nabla \widehat{v}$ is the minimizer for the relaxed problem (the fact that the minimizers are gradients is standard, as for the over-relaxed problem (6), see Section IX.3 of [13]) then the set

$$
\left\{(u, \mathbf{b}) \in A \mid K((u, \mathbf{b}), \widehat{v}) \leq E_{r e l}^{g}\left(Q_{L}\right)\right\}
$$

is not empty (take the pair $\left(\widehat{\mathbf{b}} \cdot \mathbf{e}_{d}, \widehat{\mathbf{b}}\right)$ ). It is also weakly compact, by the weak lower semi continuity of $K$ in the variable in the variable $(u, \mathbf{b})$ and the condition $u \in[-1,1]$. Thus, by the aforementioned proposition, we have

$$
\inf _{(u, \mathbf{b}) \in A} \sup _{v \in \mathrm{H}^{1}\left(Q_{L}\right)} K((u, \mathbf{b}), v)=\sup _{v \in \mathrm{H}^{1}\left(Q_{L}\right)} \inf _{(u, \mathbf{b}) \in A} K((u, \mathbf{b}), v) .
$$

Furthermore, as $|\mathbf{b}|^{2}+2 \mathbf{b} \cdot \nabla v=|\mathbf{b}+\nabla v|^{2}-|\nabla v|^{2}$, the infimum is reached for $\mathbf{b}=-\nabla v$ in $Q_{L}$ and $u=-\operatorname{sgn} v$ on $\underline{Q_{L}}$, thus

$$
\begin{aligned}
& E_{r e l}^{g}\left(Q_{L}\right)= \\
& \sup _{v} \inf _{\mathbf{b}, u}\left\{\int_{Q_{L}}\left(\frac{1}{2}|\mathbf{b}|^{2}+\mathbf{b} \cdot \nabla v\right)+\int_{\underline{Q_{L}}} v u+\int_{\Gamma_{L}} v g \mid \begin{array}{lll}
\mathbf{b} & \in & \mathrm{L}^{2}\left(Q_{L}, \mathbb{R}^{d}\right), \\
v & \in & \mathrm{L}^{2}\left(\frac{Q_{L},}{\mathrm{H}^{1}}(-1,1]\right), \\
\left.Q_{L}\right)
\end{array}\right\} \\
&=\sup _{v}\left\{-\int_{Q_{L}} \frac{1}{2}|\nabla v|^{2}-\int_{\underline{Q_{L}}}|v|+\int_{\Gamma_{L}} v g \mid v \in \mathrm{H}^{1}\left(Q_{L}\right)\right\} \\
&=-\inf _{v}\left\{\int_{Q_{L}} \frac{1}{2}|\nabla v|^{2}+\int_{\underline{Q_{L}}}|v|-\int_{\Gamma_{L}} v g \mid v \in \mathrm{H}^{1}\left(Q_{L}\right)\right\} .
\end{aligned}
$$


Proof of Lemma 9. By scaling and translation invariance, it is sufficient to consider the cube $(0,1)^{d}$. Let $w$ be a smooth function on this cube $(0,1)^{d}$, clearly

$$
\left|w\left(x^{\prime}, x_{d}\right)\right| \leq \int_{0}^{x_{d}}\left|\nabla w\left(x^{\prime}, t\right)\right| \mathrm{d} t+\left|w\left(x^{\prime}, 0\right)\right| .
$$

Integrating over $x_{d}$ between 0 and 1 , we get

$$
\int_{0}^{1}\left|w\left(x^{\prime}, x_{d}\right)\right| \mathrm{d} x_{d} \leq \int_{0}^{1}\left|\nabla w\left(x^{\prime}, t\right)\right| \mathrm{d} t+\left|w\left(x^{\prime}, 0\right)\right| .
$$

Integrating over $x^{\prime} \in(0,1)^{d-1}$ and using Hölder's inequality on the first term on the right hand side now yields

$$
\int_{(0,1)^{d}}|w| \leq\left(\int_{(0,1)^{d}}|\nabla w|^{2}\right)^{\frac{1}{2}}+\int_{(0,1)^{d-1} \times\{0\}}|w| .
$$

Next, from Lemma 3.2 in [2], with $\epsilon:=1$ we know that

$$
\int_{(0,1)^{d-1} \times\{0\}} w^{2} \lesssim \int_{(0,1)^{d}}|\nabla w|^{2}+\left(\int_{(0,1)^{d}}|w|\right)^{2},
$$

which, combined with the square of the previous estimate yields

$$
\int_{(0,1)^{d-1} \times\{0\}} w^{2} \lesssim \int_{(0,1)^{d}}|\nabla w|^{2}+\left(\int_{(0,1)^{d-1} \times\{0\}}|w|\right)^{2} .
$$

By approximation and trace estimate, (42) remains true for any $w \in \mathrm{H}^{1}\left(Q_{L}\right)$.

Now given a function $w$ on $(0,1)^{d}$ and $\epsilon \in(0,1]$, with $\epsilon=1 / N$ for some positive integer $N$, we divide the lower side of the cube into $N^{d-1}$ boxes of side length $\epsilon$. In each of these boxes (letting $x^{i}$ correspond to the centers of their bases) inequality (42) is applied to the rescaled potential $w\left(x^{i}+\epsilon x\right)$. Using the fact that the sum of squares of non-negative numbers is smaller than or equal to the square of their sum, we obtain

$$
\int_{(0,1)^{d-1} \times\{0\}} w^{2} \lesssim \epsilon \int_{(0,1)^{d-1} \times(0, \epsilon)}|\nabla w|^{2}+\frac{1}{\epsilon^{d-1}}\left(\int_{(0,1)^{d-1} \times\{0\}}|w|\right)^{2} .
$$

Extending the first integral on the right hand side to the whole of $(0,1)^{d}$ and taking the square root, we get the desired estimate. It is easily seen that up to a change of constant, this estimate holds for any $\epsilon \in(0,1]$ and not just for inverses of positive integers.

Proof of Lemma 10. A similar result is established by a somewhat different argument in [22, Remark 5.5]. We start with a couple of reductions. By scaling invariance, it is enough to prove the estimate with $(0, L)^{d}$ replaced by $(0, \pi)^{d}$. Decomposing the harmonic function $v$ into $2^{d}-1$ parts, we may restrict ourselves to the situation where $v$ has zero boundary flux on all but two of the $2^{d}$ faces of the cube $(0, \pi)^{d}$, which we call input face and output face, and zero Dirichlet boundary condition on the output face (the one on which we want to estimate the $\mathrm{L}^{2}$-norm of the normal derivative). We suppose $\mathrm{L}^{2}$-control of the normal derivative on the input face. We distinguish two cases: The easier case in which the input face is opposite to the output face and the harder case in which they are 
adjacent. By cubic symmetry, we may in both cases take the input face to be the top face $\left\{x_{d}=\pi\right\}$. In the easy case, we have

(43) $v\left(x^{\prime}, 0\right)=0$ for $x^{\prime} \in(0, \pi)^{d-1}$ and $\partial_{i} v(x)=0$ if $x_{i} \in\{0, \pi\}$ for some $i=1, \ldots, d-1$.

We then seek the following estimate on the bottom face $\left\{x_{d}=0\right\}$ :

$$
\int_{(0, \pi)^{d-1}}\left(\partial_{d} v\right)^{2}\left(x_{d}=0\right) d x_{1} \cdots d x_{d-1} \lesssim \int_{(0, \pi)^{d-1}}\left(\partial_{d} v\right)^{2}\left(x_{d}=\pi\right) d x_{1} \cdots d x_{d-1} .
$$

In the hard case, we can suppose that $v$ vanishes on the face $\left\{x_{1}=0\right\}$, we thus have

(45) $v\left(0, x_{2}, \ldots, x_{d}\right)=0$ and $\partial_{i} v(x)=0$ if $\left\{\begin{array}{l}x_{i} \in\{0, \pi\} \text { for some } i=2, \ldots, d-1, \\ \text { or } x_{1}=\pi, \\ \text { or } x_{d}=0 .\end{array}\right.$

We then seek the estimate

$$
\int_{(0, \pi)^{d-1}}\left(\partial_{1} v\right)^{2}\left(x_{1}=0\right) d x_{2} \cdots d x_{d} \lesssim \int_{(0, \pi)^{d-1}}\left(\partial_{d} v\right)^{2}\left(x_{d}=\pi\right) d x_{1} \cdots d x_{d-1} .
$$

We will show both with help of Fourier series. In the easy case, in view of (43), we may develop $v$ in Fourier series in the horizontal variables $x^{\prime}=\left(x_{1}, \ldots, x_{d-1}\right)$; because of the harmonicity of $v$ and of the fact that $v\left(\left\{x_{d}=0\right\}\right)=0$, these take on the form

$$
v=\sum_{n^{\prime}} a_{n^{\prime}} \cos \left(n_{1} x_{1}\right) \cdots \cos \left(n_{d-1} x_{d-1}\right) \sinh \left(\left|n^{\prime}\right| x_{d}\right),
$$

where the sum runs over all $n^{\prime} \in \mathbb{N}^{d-1}$ and $\left|n^{\prime}\right|^{2}=n_{1}^{2}+\cdots+n_{d-1}^{2}$. Because of

$$
\partial_{d} v=\sum_{n^{\prime}}\left|n^{\prime}\right| a_{n^{\prime}} \cos \left(n_{1} x_{1}\right) \cdots \cos \left(n_{d-1} x_{d-1}\right) \cosh \left(\left|n^{\prime}\right| x_{d}\right)
$$

and Parseval's identity we may re-express (44) as

$$
\sum_{n^{\prime}}\left|n^{\prime}\right|^{2} a_{n^{\prime}}^{2} \lesssim \sum_{n^{\prime}}\left|n^{\prime}\right|^{2} \cosh ^{2}\left(\pi\left|n^{\prime}\right|\right) a_{n^{\prime}}^{2}
$$

As $\cosh \geq 1$, this holds and the easy case (44) follows.

In the hard case, by (45), reflecting $v$ evenly across the plane $\left\{x_{1}=\pi\right\}$, we can write

$$
v=\sum_{n^{\prime}} a_{n^{\prime}} \sin \left(\left(n_{1}+\frac{1}{2}\right) x_{1}\right) \cos \left(n_{2} x_{2}\right) \cdots \cos \left(n_{d-1} x_{d-1}\right) \cosh \left(\alpha\left(n^{\prime}\right) x_{d}\right),
$$

where $\alpha\left(n^{\prime}\right)>0$ satisfies $\alpha\left(n^{\prime}\right)^{2}=\left(n_{1}+\frac{1}{2}\right)^{2}+n_{2}^{2}+\cdots+n_{d-1}^{2}$, and the sum runs over all $n^{\prime}=\left(n_{1}, \ldots, n_{d-1}\right) \in \mathbb{N}^{d-1}$. By Parseval's identity applied to the variables $x_{2}, \ldots, x_{d-1}$ over $(0, \pi)$ and to the variable $x_{1}$ over $(0,2 \pi)$ we may re-express the r. h. s. of (46) as

$$
\begin{aligned}
\int_{(0, \pi)^{d-1}}\left(\partial_{d} v\right)^{2}\left(x_{d}=\pi\right) d x_{1} \cdots d x_{d-1} & \sim \sum_{n^{\prime}} \alpha\left(n^{\prime}\right)^{2} a_{n^{\prime}}^{2} \sinh ^{2}\left(\pi \alpha\left(n^{\prime}\right)\right) \\
& \sim \sum_{n^{\prime}} \alpha\left(n^{\prime}\right)^{2} a_{n^{\prime}}^{2} \exp \left(2 \pi \alpha\left(n^{\prime}\right)\right),
\end{aligned}
$$


where the last comparison follows from the fact that $\alpha\left(n^{\prime}\right) \geq \frac{1}{2}$. For the 1. h. s. of (46), Parseval's identity applied to the variables $x_{2}, \ldots, x_{d-1}$ yields

$$
\int_{(0, \pi)^{d-1}}\left(\partial_{1} v\right)^{2}\left(x_{1}=0\right) d x_{2} \cdots d x_{d} \sim \sum_{n_{2}, \ldots, n_{d-1}} \int_{0}^{\pi}\left(\sum_{n_{1}}\left(n_{1}+\frac{1}{2}\right) a_{n^{\prime}} \cosh \left(\alpha\left(n^{\prime}\right) x_{d}\right)\right)^{2} d x_{d} .
$$

We consider the individual terms on the r. h. s. and start by expanding the square

$$
\begin{aligned}
\int_{0}^{\pi}\left(\sum _ { n _ { 1 } } \left(n_{1}+\right.\right. & \left.\left.\frac{1}{2}\right) a_{n^{\prime}} \cosh \left(\alpha\left(n^{\prime}\right) x_{d}\right)\right)^{2} d x_{d} \\
& =\sum_{n_{1}} \sum_{m_{1}}\left(n_{1}+\frac{1}{2}\right) a_{n^{\prime}}\left(m_{1}+\frac{1}{2}\right) a_{m^{\prime}} \int_{0}^{\pi} \cosh \left(\alpha\left(n^{\prime}\right) x_{d}\right) \cosh \left(\alpha\left(m^{\prime}\right) x_{d}\right) d x_{d}
\end{aligned}
$$

where, with a slight abuse of notation, $m^{\prime}:=\left(m_{1}, n_{2}, \ldots, n_{d-1}\right)$. Because of the elementary inequality

$$
\begin{aligned}
\int_{0}^{\pi} \cosh \left(\alpha\left(n^{\prime}\right) x_{d}\right) \cosh \left(\alpha\left(m^{\prime}\right) x_{d}\right) d x_{d} & \leq \int_{0}^{\pi} \exp \left(\alpha\left(n^{\prime}\right) x_{d}+\alpha\left(m^{\prime}\right) x_{d}\right) d x_{d} \\
& \leq \frac{1}{\alpha\left(n^{\prime}\right)+\alpha\left(m^{\prime}\right)} \exp \left(\pi\left(\alpha\left(n^{\prime}\right)+\alpha\left(m^{\prime}\right)\right)\right)
\end{aligned}
$$

and thus

$$
\begin{aligned}
\int_{(0, \pi)^{d-1}}\left(\partial_{1} v\right)^{2}\left(x_{1}=0\right) d x_{2} \cdots d x_{d} \\
\quad \lesssim \sum_{n_{2}, \ldots, n_{d-1}} \sum_{n_{1}} \sum_{m_{1}}\left(n_{1}+\frac{1}{2}\right)\left|a_{n^{\prime}}\right|\left(m_{1}+\frac{1}{2}\right)\left|a_{m^{\prime}}\right| \frac{1}{\alpha\left(n^{\prime}\right)+\alpha\left(m^{\prime}\right)} \exp \left(\pi\left(\alpha\left(n^{\prime}\right)+\alpha\left(m^{\prime}\right)\right)\right) \\
\leq \sum_{n_{2}, \ldots, n_{d-1}} \sum_{n_{1}} \sum_{m_{1}} \frac{1}{n_{1}+m_{1}+1} \alpha\left(n^{\prime}\right)\left|a_{n^{\prime}}\right| \exp \left(\pi \alpha\left(n^{\prime}\right)\right) \alpha\left(m^{\prime}\right)\left|a_{m^{\prime}}\right| \exp \left(\pi \alpha\left(m^{\prime}\right)\right),
\end{aligned}
$$

as $\alpha\left(n^{\prime}\right) \geq n_{1}+\frac{1}{2}$. A glance at (47) now shows that (46) reduces to the following statement on non-negative sequences $\left\{b_{n^{\prime}}:=\alpha\left(n^{\prime}\right) \exp \left(\pi \alpha\left(n^{\prime}\right)\right)\left|a_{n^{\prime}}\right|\right\}_{n^{\prime}}$

$$
\sum_{n_{2}, \ldots, n_{d-1}} \sum_{n_{1}} \sum_{m_{1}} \frac{1}{n_{1}+m_{1}+1} b_{n^{\prime}} b_{m^{\prime}} \lesssim \sum_{n_{2}, \ldots, n_{d-1}} \sum_{n_{1}} b_{n^{\prime}}^{2}
$$

which clearly can be disintegrated into

$$
\sum_{n=0}^{\infty} \sum_{m=0}^{\infty} \frac{1}{n+m+1} b_{n} b_{m} \lesssim \sum_{n=0}^{\infty} b_{n}^{2} .
$$

We conclude the proof with an argument for (48). By symmetry in $n$ and $m$, the statement follows from

$$
\sum_{n=0}^{\infty} \sum_{m=0}^{n} \frac{1}{n+m+1} b_{n} b_{m} \lesssim \sum_{n=0}^{\infty} b_{n}^{2}
$$

and to reduces to

$$
\sum_{n=0}^{\infty} b_{n} \frac{1}{n+1} \sum_{m=0}^{n} b_{m} \lesssim \sum_{n=0}^{\infty} b_{n}^{2} .
$$


Applying the Cauchy-Schwarz inequality to the left hand side we see that (47) follows directly from

$$
\sum_{n=0}^{\infty} \frac{1}{(n+1)^{2}}\left(\sum_{m=0}^{n} b_{m}\right)^{2} \lesssim \sum_{n=0}^{\infty} b_{n}^{2} .
$$

Let us prove this last estimate. In terms of the discrete anti-derivative $B_{n}:=\sum_{m=0}^{n} b_{m}$, (49) amounts to a discrete version of Hardy's inequality and can be established in a similar way: We obtain by a discrete integration by parts

$$
\begin{aligned}
\sum_{n=0}^{N} \frac{1}{(n+1)^{2}} B_{n}^{2} & \sim \sum_{n=0}^{N} \frac{1}{(n+1)(n+2)} B_{n}^{2}=\sum_{n=0}^{N}\left(\frac{1}{n+1}-\frac{1}{n+2}\right) B_{n}^{2} \\
& \leq B_{0}^{2}+\sum_{n=1}^{N} \frac{1}{n+1}\left(B_{n}^{2}-B_{n-1}^{2}\right)=b_{0}^{2}+\sum_{n=1}^{N} \frac{1}{n+1} b_{n}\left(B_{n}+B_{n-1}\right) \\
& \leq b_{0}^{2}+\left(\sum_{n=1}^{N} b_{n}^{2} \sum_{n=1}^{N} \frac{1}{(n+1)^{2}}\left(B_{n}+B_{n-1}\right)^{2}\right)^{\frac{1}{2}} \\
& \leq b_{0}^{2}+2\left(\sum_{n=1}^{N} b_{n}^{2} \sum_{n=1}^{N} \frac{1}{(n+1)^{2}} B_{n}^{2}\right)^{\frac{1}{2}}
\end{aligned}
$$

and thus by Young's inequality $\sum_{n=0}^{N} \frac{1}{(n+1)^{2}} B_{n}^{2} \lesssim \sum_{n=0}^{N} b_{n}^{2}$, which yields (49) in the limit $N \uparrow \infty$.

Proof of Lemma 11. Without loss of generality, we may assume that $L=1$ by scaling, and work in $(0,1)^{d}$. It also suffices to prove the statement for $p=2(d-1) / d$ as the other cases follow from Jensen's inequality. By the Sobolev trace theorem [1, Theorem 5.36], (which we may use because the cube is bi-Lipschitz equivalent to a ball,) and the Poincaré inequality (we may assume that $v$ has zero average,) there holds:

$$
\left(\int_{\partial(0,1)^{d}}|v|^{\frac{2(d-1)}{d-2}}\right)^{\frac{d-2}{2(d-1)}} \lesssim\left(\int_{(0,1)^{d}}|\nabla v|^{2}\right)^{\frac{1}{2}} .
$$

Noting that $q:=2(d-1) /(d-2)$ satisfies $1 / p+1 / q=1$, by integration by parts and Hölder's inequality, we may write

$$
\int_{(0,1)^{d}}|\nabla v|^{2}=\int_{\partial(0,1)^{d}} v g \leq\left(\int_{\partial(0,1)^{d}}|v|^{q}\right)^{\frac{1}{q}}\left(\int_{\partial(0,1)^{d}}|g|^{p}\right)^{\frac{1}{p}} .
$$

Using (50) and regrouping the terms in $\nabla v$ yields the desired estimate.

Proof of Lemma 12. Considering $\mathbf{b}$ on the strip $\mathbb{R}^{d-1} \times(0, L)$, the fact that it derives from a potential $(\mathbf{b}=-\nabla \tilde{v})$ is standard (as for the over-relaxed problem (6) ), see Section IX.3 of [13]). Moreover, we have $\tilde{v}(x)=v(x)+\xi^{\prime} \cdot x^{\prime}$, where $v$ is (horizontally) periodic (with period $L$ ) on $\mathbb{R}^{d} \times(0, L)$ and $\xi^{\prime} \in \mathbb{R}^{d-1}$. The latter can be seen by noting that for any $i=1, \ldots, d-1$, the function $\tilde{v}\left(\cdot+L e_{i}\right)-\tilde{v}$ has gradient $-\mathbf{b}\left(\cdot+L \mathbf{e}_{i}\right)+\mathbf{b}$, which 
vanishes by periodicity of $\mathbf{b}$, and thus must agree with some constant $\xi_{i}$, which implies that $v(x):=\tilde{v}(x)-\xi^{\prime} \cdot x^{\prime}$, where $\xi^{\prime}:=\left(\xi_{1}, \ldots, \xi_{d-1}\right)$, is periodic. It remains to prove that $\xi^{\prime}=0$. Since replacing $\mathbf{b}$ by $\mathbf{b}-f_{[0, L)^{d-1} \times(0, L)} \mathbf{b}^{\prime}$, where $\mathbf{b}^{\prime}$ denotes the horizontal component of $\mathbf{b}$, does not affect the constraint and strictly reduces the energy unless $f_{[0, L)^{d-1} \times(0, L)} \mathbf{b}^{\prime}=0$, we learn from minimality that this average must indeed vanish. Taking the average of the identity $-\mathbf{b}^{\prime}=\nabla^{\prime} v+\xi^{\prime}$ and noting that $f_{[0, L)^{d-1} \times(0, L)} \nabla^{\prime} v=0$, because the horizontal variables run over the torus, we obtain the desired $\xi^{\prime}=0$.

The fact that $v$ can be chosen to vanish on $\left\{x_{d}=L\right\}$ follows from the free boundary condition on that face, in the same way that the over-relaxed potential $v_{0}$ of (6) vanishes on $\underline{Q_{L}}$.

Proof of Lemma 13. We first prove that $v$ is Hölder continuous on $\mathbb{R}^{d-1}$. By Corollary 1 and Lemma 15, it is clear that for $\left(x^{\prime}, x_{d}\right) \in \mathbb{R}^{d-1} \times(0, L)$, there holds

$$
\left|\mathbf{b}\left(x^{\prime}, x_{d}\right)\right| \lesssim \frac{1}{x_{d}^{1 / 2}} .
$$

Thus, considering two points $x^{\prime}, y^{\prime} \in \mathbb{R}^{d-1}$, which by horizontal periodicity we may suppose to be at distance less than $L$, we join $\left(x^{\prime}, 0\right)$ to $\left(y^{\prime}, 0\right)$ by a vertical half circle contained in $\mathbb{R}^{d-1} \times(0, L)$ of diameter $2 r=\left|x^{\prime}-y^{\prime}\right|$ and integrate $\nabla v=-\mathbf{b}$ along this curve to get

$$
\left|v\left(x^{\prime}, 0\right)-v\left(y^{\prime}, 0\right)\right| \leq \int_{0}^{\pi} \frac{1}{(r \sin s)^{1 / 2}} r \mathrm{~d} s \lesssim \sqrt{r} .
$$

Furthermore by Lemma 12, $v$ vanishes on $\mathbb{R}^{d-1} \times\{L\}$. Thus the potential $v$ solves a Dirichlet problem with Hölder- $1 / 2$ boundary conditions on $\mathbb{R}^{d-1} \times\{0, L\}$. As can be seen via the representation through the Poisson kernel (for the slab), the modulus of Hölder continuity transmits (up to a constant) from the boundary data to its harmonic extension.

$$
[v]_{\mathrm{C}^{1 / 2}\left(Q_{L}\right)} \lesssim 1
$$

Let us turn to the over-relaxed potential $v_{0}$ defined on $Q_{l}$; let $w:=v-v_{0}$. As $v_{0}=0$ on $\underline{Q_{l}}$, using the first part of the lemma, there holds $w=v$ on $\underline{Q_{l}}$ hence $[w]_{\mathrm{C}^{1 / 2}\left(Q_{l}\right)} \lesssim 1$. Furthermore, since on $\Gamma_{l}, \nabla v_{0} \cdot \nu=-\mathbf{b} \cdot \nu=\nabla v \cdot \nu$, there holds $\nabla w \cdot \nu=0$ on $\bar{\Gamma}_{l}$. We thus reflect $w$ evenly across $\Gamma_{l} \cap\left\{x_{d}=l\right\}$ to extend it harmonically onto a box of double the height. Subsequently by horizontal even reflections, we extend $w$ harmonically to the whole strip $\mathbb{R}^{d-1} \times[0,2 l]$. We control the Hölder- $1 / 2$ semi-norm of $w$ on the whole boundary. We then conclude as in the case of $v$ that $[w]_{C^{1 / 2}\left(Q_{l}\right)} \lesssim 1$, which yields $\left[v_{0}\right]_{C^{1 / 2}\left(Q_{l}\right)} \lesssim 1$ by (51) and the triangle inequality.

Proof of Lemma 14. As the potential $v_{0}$ vanishes on $Q_{l}$, its odd extension across the plane $\left\{x_{d}=0\right\}$, which we still denote by $v_{0}$, is harmonic. Setting $r:=\operatorname{dist}\left(\left(x^{\prime}, 0\right), \Gamma_{l}\right)$, we thus obtain by inner regularity theory (Theorem 2.10 in [15])

$$
\left|\partial_{d} v\left(x^{\prime}, 0\right)\right| \lesssim \frac{1}{r} \sup _{B\left(\left(x^{\prime}, 0\right), r\right)}|v| .
$$


By the Hölder continuity of Lemma 13 we obtain in particular $\left|v_{0}(x)\right| \lesssim x_{d}{ }^{1 / 2}$, so that (52) yields the desired estimate in the form of $\left|\partial_{d} v\left(x^{\prime}, 0\right)\right| \lesssim r^{-1 / 2}$.

Proof of Corollary [1. By periodicity, we may assume $x^{\prime}=0$. For $L / 2 \geq x_{d} \gg 1$, by Theorem 3 there holds

$$
\int_{Q_{2 x_{d}}}|\mathbf{b}|^{2} \lesssim x_{d}{ }^{d-1}
$$

This extends to $L \geq x_{d} \gg 1$ by reflection across $\left\{x_{d}=L\right\}$, as $v=0$ on that plane. Since $|\mathbf{b}|^{2}$ is sub-harmonic in $Q_{2 x_{d}}$, the mean value property yields

$$
\left|\mathbf{b}\left(0, x_{d}\right)\right|^{2} \lesssim \frac{1}{x_{d}{ }^{d}} \int_{Q_{2 x_{d}}}|\mathbf{b}|^{2} \lesssim \frac{1}{x_{d}}
$$

Proof of Lemma 15. Consider a minimizer $(u, \mathbf{b}) \in \mathcal{A}^{\text {per }}\left(Q_{L}\right)$. We want to control the field $\mathbf{b}\left(x^{\prime}, x_{d}\right)=-\nabla v\left(x^{\prime}, x_{d}\right)$. Without loss of generality, we assume $x^{\prime}=0$. Given $R>0$, let $B_{R}^{+}:=B(0, R) \cap\left\{x_{d}>0\right\}$ and $B_{R}^{d-1}:=B(0, R) \cap\left\{x_{d}=0\right\}$. We compare $v$ with the potential $\tilde{v}$ generated by the charges $u$ in $B_{2}^{d-1}$, which can be written as a single layer potential. We only use the representation of the gradient of $\tilde{v}$ :

$$
\nabla \tilde{v}\left(x^{\prime}, x_{d}\right)=c_{d} \int_{B_{2}^{d-1}} \frac{\left(x^{\prime}-y^{\prime}, x_{d}\right)}{\left|\left(x^{\prime}, x_{d}\right)-\left(y^{\prime}, 0\right)\right|^{d}} u\left(y^{\prime}\right) \mathrm{d} y^{\prime} .
$$

The function $w:=v-\tilde{v}$ is harmonic in $B_{2}^{+}$with zero boundary flux on $B_{2}^{d-1}$; it can thus be reflected across $B_{2}^{d-1}$ to obtain a harmonic function on $B_{2}$, which we still denote by $w$. Hence $|\nabla w|^{2}$ is sub-harmonic and there holds

$$
\sup _{x \in B_{1}^{+}}|\nabla w(x)| \lesssim\left(\int_{B_{2}}|\nabla w|^{2}\right)^{\frac{1}{2}} \lesssim\left(\int_{B_{2}^{+}}|\nabla \tilde{v}|^{2}+|\nabla v|^{2}\right)^{\frac{1}{2}} .
$$

Applying Theorem 3 to $Q_{4}$, we get $\int_{B_{2}^{+}}|\nabla v|^{2} \lesssim 1$, so that it remains to control $|\nabla \tilde{v}|^{2}$. We claim that for $\left(x^{\prime}, x_{d}\right) \in B_{2}^{+}$,

$$
\left|\nabla \tilde{v}\left(x^{\prime}, x_{d}\right)\right| \lesssim \ln \left(\frac{1}{x_{d}}\right),
$$

which first gives $\sup _{x \in B_{1}^{+}}|\nabla w(x)| \lesssim 1$ by (54) and then the statement of the lemma by the triangle inequality (in the sup-norm).

The proof of estimate (55) is elementary by the representation (53), which yields

$$
\begin{aligned}
\left|\nabla \tilde{v}\left(x^{\prime}, x_{d}\right)\right| & \lesssim \int_{B_{2}^{d-1}} \frac{1}{\left(\left|x^{\prime}-y^{\prime}\right|+x_{d}\right)^{d-1}} \mathrm{~d} y^{\prime} \lesssim \int_{B_{4}^{d-1}} \frac{1}{\left(\left|z^{\prime}\right|+x_{d}\right)^{d-1}} \mathrm{~d} z^{\prime} \\
& \lesssim \int_{0}^{4} \frac{r^{d-2}}{\left(r+x_{d}\right)^{d-1}} \mathrm{~d} r \lesssim \int_{0}^{4} \frac{1}{r+x_{d}} \mathrm{~d} r \lesssim \ln \left(\frac{1}{x_{d}}\right)
\end{aligned}
$$


Proof of Lemma 16. Suppose, as in the statement of the lemma, that $L \geq l \gg 1$, and that $(u, \mathbf{b})$ is a minimizer in $\mathcal{A}^{\text {per }}\left(Q_{L}\right)$. Let $v_{0}$ be the solution to the over-relaxed problem (6) with flux boundary data $\mathbf{b} \cdot \nu$ on $\Gamma_{l}$. It suffices to construct a candidate $\left(u^{*}, \mathbf{b}^{*}\right)$ in $\mathcal{A}^{0}\left(Q_{l}\right)$ such that

$$
\frac{E\left(u^{*}, \mathbf{b}^{*}, Q_{l}\right)}{l^{d-1}} \leq \frac{E\left(u, \mathbf{b}, Q_{l}\right)}{l^{d-1}}-\frac{1}{l^{d-1}} \int_{Q_{l}} \frac{1}{2}\left|\nabla v_{0}\right|^{2}+\frac{C}{l^{1 / 2}} .
$$

The candidate field $\mathbf{b}^{*}$ will be a controlled modification of $\mathbf{b}+\nabla v_{0}$, which has vanishing flux boundary condition on $\Gamma_{l}$. We need to modify it near $Q_{l}$, jointly with $u$ in order to obtain the right flux boundary condition on $Q_{l}$. We decompose the bottom of $Q_{l}$ into cubes $Q_{i}$ of side length $\lambda \sim 1$, such that $\lambda$ divides $l$ and is large enough (depending only on $d$, in view of an application of Lemma 19) so that on each cube $Q_{i}$ there holds

$$
E\left(u, \mathbf{b}, Q_{i}\right) \lesssim \lambda^{d-1} \text { (by Theorem 3) } \text { ) or } E\left(u, \mathbf{b}, Q_{i}\right) \leq \lambda^{d-2} \Lambda \text { for some } \Lambda \lesssim 1,
$$

as well as $\left|f_{\underline{Q_{i}}} u\right| \leq \frac{1}{2}$ (by Lemma 201), and $f_{\underline{Q_{i}}}\left|\partial_{d} v_{0}\right| \leq \frac{1}{2}$ (by Lemma 14). Indeed, the last condition is satisfied for large enough $\lambda$, as, by Lemma 14;

$$
f_{\underline{Q_{i}}}\left|\partial_{d} v_{0}\right| \lesssim f_{\underline{Q_{i}}} \operatorname{dist}\left(\left(x^{\prime}, 0\right), \Gamma_{l}\right)^{-\frac{1}{2}} \mathrm{~d} x^{\prime} \lesssim \frac{1}{\lambda^{1 / 2}}
$$

For each cube $Q_{i}$ not adjacent to $\Gamma_{l}$, again by Lemma 14, we have:

$$
f_{\underline{Q_{i}}}\left|\partial_{d} v_{0}\right| \lesssim \operatorname{dist}\left(Q_{i}, \Gamma_{l}\right)^{-\frac{1}{2}}
$$

Thus, if $m_{0}$ is given by Lemma 19 with the constant $\Lambda$ coming from condition (57) (which in particular controls $\left.f_{\underline{Q_{i}}}|\nabla u|\right)$ there exists a distance $R \lesssim 1$ such that if $\operatorname{dist}\left(Q_{i}, \Gamma_{l}\right) \geq R$, then

$$
f_{\underline{Q_{i}}}\left|\partial_{d} v_{0}\right| \leq m_{0}
$$

We call these cubes "inner cubes" and treat separately the cubes with $\operatorname{dist}\left(Q_{i}, \Gamma_{l}\right)<R$ ("outer cubes"). If $Q_{i}$ is an inner cube, thanks to (59), we may apply Lemma 19 on $\underline{Q_{i}}$ with

$$
m_{i}:=f_{\underline{Q_{i}}} \partial_{d} v_{0}
$$

This yields $\tilde{u}_{i} \in\{-1,1\}$ with $\left(\tilde{u}_{i}-u\right)$ compactly supported in $\underline{Q_{i}}$ and

$$
\begin{aligned}
f_{\underline{Q_{i}}} \tilde{u}_{i} & =f_{\underline{Q_{i}}}\left(u+\partial_{d} v_{0}\right)=-\frac{1}{\lambda^{d-1}} \int_{\Gamma_{i}}\left(\mathbf{b}+\nabla v_{0}\right) \cdot \nu, \\
f_{\underline{Q_{i}}}\left|\tilde{u}_{i}-u\right| & \lesssim\left|m_{i}\right| \stackrel{(58)}{\lesssim} \operatorname{dist}\left(Q_{i}, \Gamma_{l}\right)^{-\frac{1}{2}}, \\
\int_{\underline{Q_{i}}}\left|\nabla \tilde{u}_{i}\right|-\int_{\underline{Q_{i}}}|\nabla u| & \lesssim\left|m_{i}\right| \stackrel{(58)}{\lesssim} \operatorname{dist}\left(Q_{i}, \Gamma_{l}\right)^{-\frac{1}{2}} .
\end{aligned}
$$

As $\tilde{u}_{i}=u$ near the $(d-2)$-dimensional boundary of $Q_{i}$, there is no added interface at the junction between the inner cubes. We then introduce a harmonic building block $\mathbf{b}_{i}$ 
in $Q_{i}$ with zero boundary flux on $\Gamma_{i}$ and flux boundary data $\tilde{u}_{i}-u-\partial_{d} v_{0}$ on $Q_{i}$. By Lemma 11 for $p=2$, which we may apply because of (60), the corresponding energy is controlled as

$$
\int_{Q_{i}}\left|\mathbf{b}_{i}\right|^{2} \lesssim \lambda \int_{\underline{Q_{i}}}\left|\tilde{u}_{i}-u-\partial_{d} v_{0}\right|^{2} \lesssim \int_{\underline{Q_{i}}}\left|\tilde{u}_{i}-u\right|^{2}+\int_{\underline{Q_{i}}}\left|\partial_{d} v_{0}\right|^{2} \stackrel{(61),(58)}{\lesssim} \operatorname{dist}\left(Q_{i}, \Gamma_{l}\right)^{-\frac{1}{2}}
$$

It remains to treat the outer cubes; in this case, we can modify the field more crudely as there are only about $l^{d-2}$ such cubes. If $Q_{i}$ is an outer cube, partition $Q_{i}$ into two boxes, and define $\tilde{u}_{i}$ on $Q_{i}$ such that $\tilde{u}_{i}=1$ on one box and $\tilde{u}_{i}=-1$ on the other. The size of the two boxes is chosen so that

$$
\int_{\underline{Q_{i}}}\left(\tilde{u}_{i}-\partial_{d} v_{0}-u\right)=0 .
$$

The interfacial energy in $\underline{Q_{i}}$ is given by

$$
\int_{\underline{Q_{i}}}\left|\nabla \tilde{u}_{i}\right|=\lambda^{d-2} \lesssim 1
$$

We then add a harmonic building block $\mathbf{b}_{i}$ with zero boundary flux on $\Gamma_{i}$ and flux boundary data $-\partial_{d} v_{0}-u+\tilde{u}_{i}$ on $\underline{Q_{i}}$. Fix an exponent $p<2$ for which Lemma 11 holds, it is applicable because of (63). The energy of $\mathbf{b}_{i}$ is controlled as

$$
\int_{Q_{i}}\left|\mathbf{b}_{i}\right|^{2} \lesssim \lambda^{d-(d-1) \frac{2}{p}}\left(\int_{\underline{Q_{i}}}\left|\partial_{d} v_{0}-u+\tilde{u}_{i}\right|^{p}\right)^{\frac{2}{p}} \lesssim\left(\left(\int_{\underline{Q_{i}}}\left|\partial_{d} v_{0}\right|^{p}\right)+1\right)^{\frac{2}{p}} .
$$

By Lemma 14 and since $p<2$, we obtain

$$
\int_{Q_{i}}\left|\mathbf{b}_{i}\right|^{2} \lesssim 1
$$

We note that the total interfacial energy of the union of the outer cubes is controlled by $l^{d-2}$.

We thus define a candidate in $\left(\mathbf{b}^{*}, u^{*}\right) \in \mathcal{A}^{0}\left(Q_{l}\right)$ by

$$
\begin{aligned}
& \mathbf{b}^{*}= \begin{cases}\mathbf{b}+\nabla v_{0} & \text { if } x_{d} \geq \lambda, \\
\mathbf{b}+\nabla v_{0}+\mathbf{b}_{i} & \text { in } Q_{i},\end{cases} \\
& u^{*}=\tilde{u}_{i} \text { in } \underline{Q_{i}} .
\end{aligned}
$$

To compute its total energy $E\left(\mathbf{b}^{*}, u^{*}, Q_{l}\right)$, we start with the interfacial energy

$$
\begin{aligned}
\int_{\underline{Q_{l}}}\left|\nabla u^{*}\right| & \stackrel{\sqrt{64}}{\leq} \int_{\underline{Q_{l}}}|\nabla u|+\sum_{Q_{i} \text { inner cube }}\left(\int_{\underline{Q_{i}}}\left|\nabla \tilde{u}_{i}\right|-\int_{\underline{Q_{i}}}|\nabla u|\right)+C l^{d-2} \\
& \stackrel{(62)}{\leq} \int_{\underline{Q_{l}}}|\nabla u|+C\left(\sum_{Q_{i} \text { inner cube }} \operatorname{dist}\left(Q_{i}, \Gamma_{l}\right)^{-1}+l^{d-2}\right) \\
& \leq \int_{\underline{Q_{l}}}|\nabla u|+C l^{d-\frac{3}{2}} .
\end{aligned}
$$


For the field energy, by Lemma 7, we note

$$
\int_{Q_{l}}\left|\mathbf{b}^{*}\right|^{2} \stackrel{(66)}{=} \int_{Q_{l}}\left|\mathbf{b}+\mathbf{b}_{i} \mathbb{1}_{Q_{i}}+\nabla v_{0}\right|^{2}=\int_{Q_{l}}\left|\mathbf{b}+\mathbf{b}_{i} \mathbb{1}_{Q_{i}}\right|^{2}-\int_{Q_{l}}\left|\nabla v_{0}\right|^{2}
$$

Thus, there holds

$$
\int_{Q_{l}}\left|\mathbf{b}^{*}\right|^{2}+\int_{Q_{l}}\left|\nabla v_{0}\right|^{2} \stackrel{\sqrt[(66)]{=}}{=} \int_{Q_{l} \cap\left\{x_{d} \geq \lambda\right\}}|\mathbf{b}|^{2}+\sum_{i} \int_{Q_{i}}\left|\mathbf{b}+\mathbf{b}_{i}\right|^{2}
$$

and thus

$$
\begin{aligned}
\int_{Q_{l}}\left|\mathbf{b}^{*}\right|^{2}+ & \int_{Q_{l}}\left|\nabla v_{0}\right|^{2}-\int_{Q_{l}}|\mathbf{b}|^{2}=\sum_{i} \int_{Q_{i}}\left(\left|\mathbf{b}_{i}\right|^{2}+2 \mathbf{b} \cdot \mathbf{b}_{i}\right) \\
& \lesssim \sum_{Q_{i} \text { outer cube }} \int_{Q_{i}}\left(\left|\mathbf{b}_{i}\right|^{2}+|\mathbf{b}|^{2}\right)+\sum_{Q_{i} \text { inner cube }}\left(\int_{Q_{i}}\left|\mathbf{b}_{i}\right|^{2}+\left|\int_{Q_{i}} \mathbf{b} \cdot \mathbf{b}_{i}\right|\right) .
\end{aligned}
$$

For the inner cubes, we integrate the mixed terms by parts, using the facts that $\mathbf{b}$ is the gradient of a Hölder-1/2 potential $v$ (cf. Lemma 13) and that $\mathbf{b}_{i}$ is divergence-free with zero boundary flux on $\Gamma_{i}$. More precisely, choosing a point $\left(x_{i}^{\prime}, 0\right)$ at the bottom of the inner cube $Q_{i}$, we get:

$$
\begin{aligned}
\left|\int_{Q_{i}} \mathbf{b} \cdot \mathbf{b}_{i}\right| & =\mid \int_{\underline{Q_{i}}}\left(v-v\left(\left(x_{i}^{\prime}, 0\right)\right) \mathbf{b}_{i} \cdot \nu|=| \int_{\underline{Q_{i}}}\left(v-v\left(\left(x_{i}^{\prime}, 0\right)\right)\left(\tilde{u}_{i}-\partial_{d} v_{0}-u\right) \mid\right.\right. \\
& \leq \sup _{x^{\prime} \in \underline{Q_{i}}}\left|v\left(x^{\prime}, 0\right)-v\left(\left(x_{i}^{\prime}, 0\right)\right)\right|\left(\int_{\underline{Q_{i}}}\left|\tilde{u}_{i}-u\right|+\int_{\underline{Q_{i}}}\left|\partial_{d} v_{0}\right|\right) \\
& \stackrel{(58),(61)}{\lesssim} \operatorname{dist}\left(Q_{i}, \Gamma_{l}\right)^{-\frac{1}{2}} .
\end{aligned}
$$

For the outer cubes, we appeal to (65) and condition (57) in the choice of $\lambda$. We plug this into the estimate (68) to obtain

$$
\int_{Q_{l}}\left|\mathbf{b}^{*}\right|^{2}+\int_{Q_{l}}\left|\nabla v_{0}\right|^{2}-\int_{Q_{l}}|\mathbf{b}|^{2} \lesssim l^{d-2}+\sum_{Q_{i} \text { inner cube }} \operatorname{dist}\left(Q_{i}, \Gamma_{l}\right)^{-\frac{1}{2}} \lesssim l^{d-\frac{3}{2}}
$$

Combining interfacial and field energy, cf. (67) and the last estimate, we get the desired (56) in form of

$$
E\left(u^{*}, \mathbf{b}^{*}, Q_{l}\right) \leq E\left(u, \mathbf{b}, Q_{l}\right)-\int_{Q_{l}} \frac{1}{2}\left|\nabla v_{0}\right|^{2}+C l^{d-\frac{3}{2}}
$$

Proof of Lemma 17. Recall that $v_{0}$ is the solution to the over-relaxed problem (6) in $Q_{l}$ with flux boundary data $\mathbf{b} \cdot \nu$ on $\Gamma_{l}$. Consider a periodic minimizer $\left(u^{\text {per }}, \mathbf{b}^{\text {per }}\right) \in \mathcal{A}^{\text {per }}\left(Q_{l}\right)$. Let $v_{0}^{\text {per }}$ denote the solution to the over-relaxed problem in $Q_{l}$ with flux boundary data $\mathbf{b}^{p e r} \cdot \nu$. Notice that the field $\mathbf{b}^{p e r}+\nabla v_{0}^{p e r}-\nabla v_{0}$ has the same normal flux boundary 
conditions as $\mathbf{b}$ on $\Gamma_{l}$. We will modify $\left(u^{\text {per }}, \mathbf{b}^{\text {per }}+\nabla v_{0}^{\text {per }}-\nabla v_{0}\right)$ in order to obtain a candidate $\left(u^{*}, \mathbf{b}^{*}\right)$ in $\mathcal{A}^{\mathbf{b} \cdot \nu}\left(Q_{l}\right)$ such that

$$
\frac{E\left(u^{*}, \mathbf{b}^{*}, Q_{l}\right)}{l^{d-1}} \leq \frac{E\left(u^{p e r}, \mathbf{b}^{p e r}, Q_{l}\right)}{l^{d-1}}+\frac{1}{l^{d-1}} \int_{Q_{l}} \frac{1}{2}\left|\nabla v_{0}\right|^{2}+\frac{C}{l^{1 / 2}},
$$

which implies (12) up to an additional interfacial energy of order $l^{d-2}$. As in Lemma 16. we decompose the bottom of $Q_{l}$ into cubes $Q_{i}$ and consider separately the cubes near $\Gamma_{l}$ and the others. We choose cubes of side length $\lambda \sim 1$, where $\lambda$ divides $l$ and is such that on each cube $Q_{\lambda}$ of side length $\lambda$ contained in $Q_{l}$ and with base in $\underline{Q_{l}}$, there holds $E\left(u^{\mathrm{per}}, \mathbf{b}^{\mathrm{per}}, Q_{\lambda}\right) \lesssim 1$ ( by Theorem 3), $\left|f_{\underline{Q}_{\lambda}} u^{\mathrm{per}}\right| \leq \frac{1}{2}$ (by Lemma 201), and $f_{Q_{\lambda}}\left|\partial_{d} v_{0}^{\text {per }}\right| \leq \frac{1}{4}$ and $f_{Q_{\lambda}}\left|\partial_{d} v_{0}\right| \leq \frac{1}{4}$ (by Lemma 14). Lemma 14 in facts yields the following more precise estimate for an inner cube $Q_{i}$

$$
f_{\underline{Q_{i}}}\left(\left|\partial_{d} v_{0}^{p e r}\right|+\left|\partial_{d} v_{0}\right|\right) \lesssim \operatorname{dist}\left(Q_{i}, \Gamma_{l}\right)^{-\frac{1}{2}}
$$

We do not detail the construction of the new charge $u^{*}$ (equal to $\tilde{u}_{i}$ on the cubes $Q_{i}$ ) as it is similar to what we did in the proof of Lemma 16, replacing $u$ by $u^{\text {per }}$ when applying Lemma 19 for the inner cubes with

$$
m_{i}:=f_{\underline{Q_{i}}}\left(-\partial_{d} v_{0}^{p e r}-\partial_{d} v_{0}\right) .
$$

When it comes to the construction of $\mathbf{b}^{*}$ there is a slight difference coming from the fact that we need to take both $\nabla v_{0}$ and $\nabla v_{0}^{\text {per }}$ into account. On a cube $Q_{i}$, we let $\mathbf{b}_{i}$ be the harmonic building block corresponding to Lemma 11 with zero boundary flux on $\Gamma_{i}$ and flux boundary data $\tilde{u}_{i}-u^{\text {per }}+\partial_{d} v_{0}^{\text {per }}-\partial_{d} v_{0}$ on $\underline{Q_{i}}$. We then define $\left(u^{*}, \mathbf{b}^{*}\right) \in \mathcal{A}^{\mathbf{b} \cdot \nu}\left(Q_{L}\right)$ by

$$
\begin{aligned}
& \mathbf{b}^{*}= \begin{cases}\mathbf{b}^{\text {per }}+\nabla v_{0}^{p e r}-\nabla v_{0} & \text { if } x_{d} \geq \lambda, \\
\mathbf{b}^{\text {per }}+\nabla v_{0}^{p e r}-\nabla v_{0}+\mathbf{b}_{i} & \text { if }\left(x^{\prime}, x_{d}\right) \in Q_{i},\end{cases} \\
& u^{*}=\tilde{u}_{i} \quad \text { in } \underline{Q_{i}} .
\end{aligned}
$$

As for (67), the interfacial energy is controlled as

$$
\int_{\underline{Q_{l}}}\left|\nabla u^{*}\right| \leq \int_{\underline{Q_{l}}}\left|\nabla u^{p e r}\right|+C l^{d-\frac{3}{2}} .
$$

To compute the energy of $\mathbf{b}^{*}$, we first notice that Lemma 7 implies

$$
\begin{aligned}
\int_{Q_{l}}\left|\mathbf{b}^{*}\right|^{2}-\int_{Q_{l}}\left|\nabla v_{0}\right|^{2} & =\int_{Q_{l}}\left|\mathbf{b}^{*}+\nabla v_{0}\right|^{2} \\
& \stackrel{(700)}{=} \int_{Q_{l} \cap\left\{x_{d} \geq \lambda\right\}}\left|\mathbf{b}^{\text {per }}+\nabla v_{0}^{\text {per }}\right|^{2}+\sum_{i} \int_{Q_{i}}\left|\mathbf{b}^{\text {per }}+\nabla v_{0}^{\text {per }}+\mathbf{b}_{i}\right|^{2} \\
& =\int_{Q_{l}}\left|\mathbf{b}^{\text {per }}+\nabla v_{0}^{\text {per }}\right|^{2}+\sum_{i} \int_{Q_{i}}\left(2\left(\mathbf{b}^{\text {per }}+\nabla v_{0}^{\text {per }}\right) \cdot \mathbf{b}_{i}+\left|\mathbf{b}_{i}\right|^{2}\right) .
\end{aligned}
$$


We apply Lemma 7 to $\mathbf{b}^{\text {per }}+\nabla v_{0}^{\text {per }}$ and use the fact that $\int_{Q_{l}}\left|\nabla v_{0}^{\text {per }}\right|^{2} \geq 0$ to obtain

$$
\begin{aligned}
\int_{Q_{l}}\left|\mathbf{b}^{*}\right|^{2}-\int_{Q_{l}}\left|\nabla v_{0}\right|^{2} & -\int_{Q_{l}}\left|\mathbf{b}^{\text {per }}\right|^{2} \\
\qquad \int_{Q_{l}}\left|\nabla v_{0}^{p e r}\right|^{2} & +\sum_{Q_{i} \text { outer cube }} \int_{Q_{i}}\left(\left|\mathbf{b}_{i}\right|^{2}+\left|\mathbf{b}^{\text {per }}\right|^{2}\right) \\
& +\sum_{Q_{i} \text { inner cube }}\left(\int_{Q_{i}}\left|\mathbf{b}_{i}\right|^{2}+\left|\int_{Q_{i}} \mathbf{b}^{\text {per }} \cdot \mathbf{b}_{i}\right|\right) .
\end{aligned}
$$

Applying Lemma 16 to $\left(u^{\text {per }}, \mathbf{b}^{\text {per }}\right)$ (which is a minimizer in $\mathcal{A}^{\text {per }}\left(Q_{l}\right)$ ) and using Corollary 2 (which only relied on Lemma 16) to get $\left|\sigma^{\text {per }}\left(Q_{l}\right)-\sigma^{0}\left(Q_{l}\right)\right| \lesssim l^{-1 / 2}$, we obtain

$$
\frac{1}{l^{d-1}} \int_{Q_{l}} \frac{1}{2}\left|\nabla v_{0}^{p e r}\right|^{2} \leq \sigma^{p e r}\left(Q_{l}\right)-\sigma^{0}\left(Q_{l}\right)+\frac{C}{l^{1 / 2}} \lesssim \frac{1}{l^{1 / 2}}
$$

The remaining terms on the r. h. s. of (72) are controlled by the same arguments as those in (68), to the effect of

$$
\int_{Q_{l}}\left|\mathbf{b}^{*}\right|^{2}-\int_{Q_{l}}\left|\nabla v_{0}\right|^{2}-\int_{Q_{l}}\left|\mathbf{b}^{p e r}\right|^{2} \lesssim l^{d-\frac{3}{2}}
$$

Combined with (71), this yields (69).

Proof of Corollary Q. Using statements (vii) and (ii) of Lemma 1 as well as Lemma 16 for $l=L \gg 1$, we obtain

$$
\sigma^{\text {per }}\left(Q_{L}\right) \leq \sigma\left(Q_{L}\right)+\frac{C}{L} \leq \sigma^{0}\left(Q_{L}\right)+\frac{C}{L} \leq \sigma^{p e r}\left(Q_{L}\right)+\frac{C}{L^{1 / 2}} .
$$

Thus, it suffices to prove that there exists $\sigma^{*} \in(0,+\infty)$ with $\left|\sigma^{0}\left(Q_{L}\right)-\sigma^{*}\right| \lesssim L^{-1 / 2}$. In fact, it even suffices to prove the existence of the limit $\sigma^{*}$ of $\sigma^{0}\left(Q_{L}\right)$ and thus of $\sigma\left(Q_{L}\right)$ as $L \uparrow+\infty$. Indeed, supposing the limit exists, statements (iii), (iii), and (ii) of Lemma 11, together with (73) imply

$$
\sigma\left(Q_{L}\right) \leq \lim _{k \rightarrow \infty} \sigma\left(Q_{k L}\right)=\sigma^{*}=\lim _{k \rightarrow \infty} \sigma^{0}\left(Q_{k L}\right) \leq \sigma^{0}\left(Q_{L}\right) \leq \sigma\left(Q_{L}\right)+\frac{C}{L^{1 / 2}} .
$$

We now argue that $\lim _{L \rightarrow+\infty} \sigma^{0}\left(Q_{L}\right) \in(0,+\infty)$ by showing that the integer monotonicity of Lemma 1 (iii) approximately extends to all $L \gg l \gg 1$ in form of

$$
\sigma^{0}\left(Q_{L}\right) \leq \sigma^{0}\left(Q_{l}\right)+\frac{C}{l}
$$

This together with Lemma (1) (ii) and (v) yield the existence of a positive and finite limit. In order to prove (74), let $(u, \mathbf{b})$ be a minimizer in $\mathcal{A}^{0}\left(Q_{L}\right)$ for $L \gg 1$. Given a positive number $\lambda$, we define $\left(u^{\lambda}, \mathbf{b}^{\lambda}\right)$ by $u^{\lambda}(\lambda \cdot)=u(\cdot)$ and $\mathbf{b}^{\lambda}(\lambda \cdot)=\mathbf{b}(\cdot)$ (as in the proof of Theorem 2). Clearly $\left(u^{\lambda}, \mathbf{b}^{\lambda}\right) \in \mathcal{A}^{0}\left(Q_{\lambda L}\right)$, and it is easy to see by a change of variables that

$$
\frac{E\left(u^{\lambda}, \mathbf{b}^{\lambda}, Q_{\lambda L}\right)}{(\lambda L)^{d-1}} \leq \max \left\{\lambda, \frac{1}{\lambda}\right\} \frac{E\left(u, \mathbf{b}, Q_{L}\right)}{L^{d-1}} .
$$


From this we infer that for $L \geq L^{\prime} \gg 1, \sigma^{0}\left(Q_{L}\right) \leq\left(L / L^{\prime}\right) \sigma^{0}\left(Q_{L^{\prime}}\right)$ and in turn, as Lemma 1 (iv) implies $\sigma^{0}\left(Q_{L}\right) \lesssim 1$, there holds

$$
\left|\sigma^{0}\left(Q_{L^{\prime}}\right)-\sigma^{0}\left(Q_{L}\right)\right| \lesssim \frac{L}{L^{\prime}}-1
$$

Now, for $L \gg l \gg 1$, let $k \in \mathbb{N}$ be such that $(k+1) l>L \geq k l$; applying (75) with $L^{\prime}=k L$, we obtain by Lemma 1 (iii)

$$
\sigma^{0}\left(Q_{L}\right) \leq \sigma^{0}\left(Q_{k l}\right)+\frac{C}{l} \leq \sigma^{0}\left(Q_{l}\right)+\frac{C}{l} .
$$

Proof of Lemma 18. Let $1 \ll l^{\prime} \ll l \leq L$, where we assume $l$ to be an odd integer multiple of $l^{\prime}$. Let $-\nabla v_{0}^{l^{\prime}}$ be the solution of the over-relaxed problem (6) in $Q_{l^{\prime}}$ with flux boundary data $\mathbf{b} \cdot \nu$ on $\Gamma_{l^{\prime}}$. We first claim that

$$
\int_{Q_{l^{\prime}}}\left|\nabla v_{0}^{l^{\prime}}-\nabla v_{0}^{l}\right|^{2} \lesssim l^{d-1} l^{\prime} \frac{1}{2}
$$

To see this, we decompose $Q_{l} \cap\left\{x_{d}<l^{\prime}\right\}$ into cubes $Q^{i}$ of side $l^{\prime}$ (including $Q_{l^{\prime}}$ ), and denote by $-\nabla v_{0}^{i}$ the corresponding solutions of the over-relaxed problem. Then the field

$$
\tilde{\mathbf{b}}:=\left\{\begin{array}{cl}
-\nabla v_{0}^{i} & \text { on } Q^{i}, \\
\mathbf{b} & \text { on } Q_{l} \cap\left\{x_{d} \geq l^{\prime}\right\},
\end{array}\right.
$$

is a competitor for the over-relaxed problem on $Q_{l}$, so that, using Lemma 7 ,

$$
\begin{aligned}
\int_{Q_{l}} \frac{1}{2}\left|\tilde{\mathbf{b}}+\nabla v_{0}^{l}\right|^{2} & \leq \int_{Q_{l}} \frac{1}{2}|\tilde{\mathbf{b}}|^{2}-\int_{Q_{l}} \frac{1}{2}\left|\nabla v_{0}^{l}\right|^{2} \\
& \leq \int_{Q_{l} \cap\left\{x_{d}>l^{\prime}\right\}} \frac{1}{2}|\mathbf{b}|^{2}+\sum_{i} \int_{Q^{i}} \frac{1}{2}\left|\nabla v_{0}^{i}\right|^{2}-\int_{Q_{l}} \frac{1}{2}\left|\nabla v_{0}^{l}\right|^{2} .
\end{aligned}
$$

On the one hand, we know by Lemma 16 and Corollary 2 that

$$
\int_{Q^{i}} \frac{1}{2}\left|\nabla v_{0}^{i}\right|^{2} \leq E\left(u, \mathbf{b}, Q^{i}\right)-\sigma^{*} l^{\prime d-1}+C l^{\prime}{ }^{d-\frac{3}{2}} .
$$

On the other hand, by Lemma 17 and Corollary 2, we have

$$
-\int_{Q_{l}} \frac{1}{2}\left|\nabla v_{0}^{l}\right|^{2} \leq E\left(u, \mathbf{b}, Q_{l}\right)-\sigma^{*} l^{d-1}+C l^{d-\frac{3}{2}} .
$$

Since the energy is additive up to the interfacial energy coming from pasting,

$$
E\left(u, \mathbf{b}, Q_{l}\right) \leq \int_{Q_{l} \cap\left\{x_{d}>l^{\prime}\right\}} \frac{1}{2}|\mathbf{b}|^{2}+\sum_{i} E\left(u, \mathbf{b}, Q^{i}\right)+C \frac{l}{l^{\prime}} l^{d-2},
$$

we obtain

$$
\int_{Q_{l}} \frac{1}{2}\left|\tilde{\mathbf{b}}+\nabla v_{0}^{l}\right|^{2} \lesssim\left(\frac{l}{l^{\prime}}\right)^{d-1} l^{\prime d-\frac{3}{2}}+\frac{l}{l^{\prime}} l^{d-2} \lesssim \frac{l^{d-1}}{l^{\prime 1 / 2}} .
$$

Restricting the integral to $Q_{l^{\prime}}$, we recover (76) by definition of $\tilde{\mathbf{b}}$. 
Second, we claim

$$
\int_{Q_{l^{\prime}}}\left|\nabla v_{0}^{l}\right|^{2} \lesssim\left(\frac{l^{\prime}}{l}\right)^{d} \int_{Q_{l}}\left|\nabla v_{0}^{l}\right|^{2} .
$$

Indeed, reflecting $v_{0}^{l}$ oddly across $\left\{x_{d}=0\right\}$, we obtain a harmonic function on the box $(-l / 2, l / 2)^{d-1} \times(-l, l)$, so that (77) is a consequence of the mean-value property of the sub-harmonic function $\left|\nabla v_{0}^{l}\right|^{2}$.

Combining (76) and (77) with the triangle inequality, and letting $\theta:=l^{\prime} / l$ we get

$$
\frac{1}{(\theta l)^{d-1}} \int_{Q_{\theta l}} \frac{1}{2}\left|\nabla v_{0}^{\theta l}\right|^{2} \leq C_{0}\left(\theta \frac{1}{l^{d-1}} \int_{Q_{l}} \frac{1}{2}\left|\nabla v_{0}^{l}\right|^{2}+\frac{1}{(\theta l)^{1 / 2}}\right) .
$$

We fix $\theta$, depending only on $d$, such that $1 / \theta$ is an odd integer, and so small that $C_{0} \theta \leq 1 / 2$; so that letting $D_{l}:=l^{-(d-1)} \int_{Q_{l}} \frac{1}{2}\left|\nabla v_{0}^{l}\right|^{2}$, we obtain

$$
D_{\theta l} \leq \frac{1}{2} D_{l}+\frac{C}{l^{1 / 2}} .
$$

Applying Lemma 16 and Corollary 2 to $l=L$, we get

$$
D_{L} \leq \frac{E\left(u, \mathbf{b}, Q_{L}\right)}{L^{d-1}}-\sigma^{0}\left(Q_{L}\right)+\frac{C}{L^{1 / 2}} \lesssim \frac{1}{L^{1 / 2}} .
$$

With this anchoring, we iterate (178), to obtain the desired $D_{l} \lesssim l^{-1 / 2}$ for $l \gg 1$.

Proof of Lemma [19. By rescaling, we may assume without loss of generality that $\lambda=1$, so that $\underline{Q}=(0,1)^{d-1}$. We will define a smooth vector field $\xi$, compactly supported in $\underline{Q}$, such that $\nabla \cdot \xi$ approximates $u-f_{Q} u$. Then we will transport the values of $u$ along the flow generated by $\xi$, for positive or negative time depending on whether we want to increase or decrease the average charge. The flow of $\xi$ is the map $\Phi .(\cdot):(t, x) \in \mathbb{R} \times \underline{Q} \rightarrow \underline{Q}$ solving the differential equation

$$
\left\{\begin{aligned}
\partial_{t} \Phi_{t}(x) & =\xi\left(\Phi_{t}(x)\right) & & \text { for }(t, x) \in \mathbb{R} \times \underline{Q}, \\
\left.\Phi_{t}\right|_{t=0} & =\Phi_{0}=\mathrm{id} & & \text { on } \underline{Q} .
\end{aligned}\right.
$$

As $\xi$ will be smooth and compactly supported, for all $t \in \mathbb{R}, \Phi_{t}$ is a diffeomorphism of $\underline{Q}$ that coincides with the identity close to the boundary of $\underline{Q}$. For shortness, we set

$$
u_{t}:=u \circ \Phi_{t}^{-1} \text {. }
$$

By definition, $u_{t}$, like $u$, takes values into $\{-1,1\}$. We shall construct $\xi$ such that

$$
\frac{d}{d t} \int_{\underline{Q}} u_{t} \geq \frac{1}{4} \quad \text { for }|t| \ll 1
$$

and that $\int_{\underline{Q}}\left|u_{t}-u\right|$ and $\int_{\underline{Q}}\left|\nabla u_{t}\right|$ are Lipschitz continuous at $t=0$. This will allow us to define $\tilde{u}:=u_{t}$ for some appropriate $t \in[-4 m, 4 m]$ with the desired properties.

We now turn to the construction of the vector field $\xi$. In order to flow from positive to negative charges, we want $\nabla \cdot \xi \simeq u-f u$. In particular, a good candidate is the gradient 
of the potential $\psi$ obtained by solving the following Poisson problem

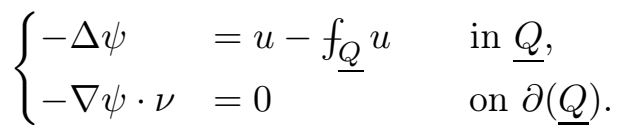

Notice that the vector field $-\nabla \psi$ has divergence equal to $u-f_{Q} u$. We reflect $u$ and $\psi$ evenly along the sides of $\underline{Q}$ to extend them to the whole of $\mathbb{R}^{d-\overline{1}}$. The extended $\psi$ still satisfies $-\Delta \psi=u-f_{\underline{Q}} u$. Since $\sup _{\mathbb{R}^{d-1}}\left|u-f_{\underline{Q}} u\right| \leq 2$, by elliptic regularity (we refer to Section 8.11 in [15]), $\bar{\nabla} \psi$ is Hölder-1/2 continuous. In particular, it is square integrable in the larger cube $\underline{\tilde{Q}}:=(-1,2)^{d-1}$. We will use the following uniform bounds:

$$
\forall x, y \in \mathbb{R}^{d-1},|\nabla \psi(y)-\nabla \psi(x)| \lesssim|y-x|^{\frac{1}{2}} \quad \text { and } \quad \int_{\underline{\tilde{Q}}}|\nabla \psi|^{2} \lesssim 1 .
$$

To obtain $\xi$, we cut off and mollify $-\nabla \psi$ to obtain a smooth vector field, compactly supported in $Q$. Given $r \in(0,1 / 2]$, for any function $f$, we denote by $f^{r}:=f * \varphi_{r}$ the convolution of $f$ with a standard mollifier $\varphi_{r}$ on the scale $r$. Next, fix $\eta_{r}^{1}:(0,1) \rightarrow[0,1]$ to be a smooth compactly supported cut-off function such that $\eta_{r}^{1}(s)=1$ for $s \in[r, 1-r]$ , and $\left|\left(\eta_{r}^{1}\right)^{\prime}\right| \lesssim \frac{1}{r}$. We now define a cut-off function $\eta_{r}$ on $\underline{Q}$ for $x=\left(x_{1}, \ldots, x_{d-1}\right) \in \underline{Q}$ by $\eta_{r}(x):=\prod_{i=1}^{d-1} \eta_{r}^{1}\left(x_{i}\right)$. Finally we define $\xi$ on $\underline{Q}$ by

$$
\xi:=-\eta_{r} \nabla \psi^{r} \text {. }
$$

We do not stress the dependence of $\xi$ on $r$, as $r$ will later be fixed. There holds

$$
\nabla \cdot \xi=-\eta_{r} \Delta \psi^{r}-\nabla \eta_{r} \cdot \nabla \psi^{r} \stackrel{(800)}{=} \eta_{r}\left(u^{r}-f_{\underline{Q}} u\right)-\nabla \eta_{r} \cdot \nabla \psi^{r} .
$$

Let us show that the second term $\nabla \eta_{r} \cdot \nabla \psi^{r}$ is small in the $\mathrm{L}^{1}$-norm, for small $r$. Notice that $\nabla \eta_{r}$ is supported in the set of points lying at distance less than $r$ to $\partial(\underline{Q})$. Consider one of these points $x$. Without loss of generality, we may suppose that there exists $k \in\{1, \ldots, d-2\}$ such that $x_{i} \in(0, r)$ for $i \leq k$ and $x_{i} \in[r, 1-r]$ for $i>k$. As $x_{i} \in[r, 1-r]$ implies $\left(\eta_{r}^{1}\right)^{\prime}\left(x_{i}\right)=0$, there holds

$$
\nabla \eta_{r} \cdot \nabla \psi^{r}(x)=\sum_{i=1}^{k} \partial_{i} \eta_{r}(x) \partial_{i} \psi^{r}(x) .
$$

By estimate (81) and the Neumann boundary condition in (80), for $i \leq k$, we have

$$
\left|\partial_{i} \psi^{r}(x)\right| \lesssim r^{\frac{1}{2}}
$$

Together with $\left|\nabla \eta_{r}\right| \lesssim 1 / r$, we thus obtain

$$
\left|\nabla \eta_{r}(x) \cdot \nabla \psi^{r}(x)\right| \lesssim \frac{1}{r^{1 / 2}}
$$

The set of all such $x$ has area of order $r$. We thus have

$$
\int_{\underline{Q}}\left|\nabla \eta_{r} \cdot \nabla \psi^{r}\right| \lesssim r^{\frac{1}{2}}
$$


On the one hand, by the convolution estimate and recalling the extension of $u$ by even reflection,

$$
\int_{\underline{Q}}\left|u^{r}-u\right| \lesssim r \int_{\underline{Q}}|\nabla u| \leq r \Lambda
$$

and on the other hand, as $|u| \leq 1$,

$$
\int_{\underline{Q}}\left|\left(\eta_{r}-1\right)\left(u^{r}-f_{\underline{Q}} u\right)\right| \lesssim r .
$$

We infer

$$
\begin{aligned}
& \int_{\underline{Q}}\left|\nabla \cdot \xi-\left(u-f_{\underline{Q}} u\right)\right| \stackrel{\sqrt[833]{\leq}}{\leq} \int_{\underline{Q}}\left|\eta_{r}\left(u^{r}-f_{\underline{Q}} u\right)-\nabla \eta_{r} \cdot \nabla \psi^{r}-\left(u-f_{\underline{Q}} u\right)\right| \\
& \text { (87) } \quad \leq \int_{\underline{Q}}\left|u^{r}-u\right|+\int_{\underline{Q}}\left|\left(\eta_{r}-1\right)\left(u^{r}-f_{\underline{Q}} u\right)\right|+\int_{\underline{Q}}\left|\nabla \eta_{r} \cdot \nabla \psi^{r}\right| \stackrel{(85),(866),(84)}{\lesssim \Lambda} r^{\frac{1}{2}} .
\end{aligned}
$$

Now that we have this control over $\xi$, let us prove that its flow modifies the global charge in the desired way (79). By a change of variables,

$$
\int_{\underline{Q}} u_{t}=\int_{\underline{Q}} u \operatorname{det} \mathrm{D} \Phi_{t} .
$$

Using Liouville's formula to differentiate the determinant and operating the converse change of variables yields

$$
\frac{d}{d t} \int_{\underline{Q}} u_{t}=\int_{\underline{Q}} u(\nabla \cdot \xi) \circ \Phi_{t} \operatorname{det} \mathrm{D} \Phi_{t}=\int_{\underline{Q}} u_{t} \nabla \cdot \xi .
$$

Hence at $t=0$, using (87) and the fact that $|u|=1$, we obtain

$$
\left|\frac{d}{d t}\right|_{t=0} \int_{\underline{Q}} u_{t}-\int_{\underline{Q}} u\left(u-f_{\underline{Q}} u\right) \mid \lesssim_{\Lambda} r^{\frac{1}{2}},
$$

so together with the assumption (13), we get

$$
\frac{3}{4}-\left.\frac{d}{d t}\right|_{t=0} \int_{\underline{Q}} u_{t} \lesssim \Lambda r^{\frac{1}{2}} .
$$

Hence we now may fix $r>0$ so small that

$$
\left.\frac{d}{d t}\right|_{t=0} \int_{\underline{Q}} u_{t} \geq \frac{1}{2} .
$$

It remains to prove that (79) holds also for $t$ small enough and not just at $t=0$. We postpone this and start by proving that the total variation of $u_{t}$ is uniformly bounded. By [21, Theorem 17.5], or by a standard generalization of [17, Theorem 10.4], the first variation of the total variation of $u_{t}$ at time $t$ along the flow of $\xi$ is equal to

$$
\frac{d}{d t} \int_{\underline{Q}}\left|\nabla u_{t}\right|=-\int_{\underline{Q}}\left(\nabla_{\tan } \cdot \xi\right)\left|\nabla u_{t}\right|
$$


where $\nabla_{\tan } \cdot \xi$ is the tangential divergence of $\xi$ along the reduced boundary of the set of finite perimeter $\left\{u_{t}=-1\right\}$. Since $r$ is now fixed and depends only and $\Lambda$ and $d$, we obtain from (82), the fact that $\psi^{r}=\psi * \varphi_{r}$ and the Hölder inequality that

$$
\sup _{\underline{Q}}|\xi| \lesssim \Lambda \quad\left(\sup _{\underline{Q}}|\nabla \psi|^{2}\right)^{\frac{1}{2}} \underset{\lesssim \Lambda}{\stackrel{\sqrt[810]{ }}{\lesssim}} 1 \text { and } \sup _{\underline{Q}}|\mathrm{D} \xi| \lesssim \Lambda 1 .
$$

Hence

and thus for $t \in[-1,1]$

$$
\left|\frac{d}{d t} \int_{\underline{Q}}\right| \nabla u_{t}|| \lesssim \Lambda \int_{\underline{Q}}\left|\nabla u_{t}\right|
$$

$$
\int_{\underline{Q}}\left|\nabla u_{t}\right|-\int_{\underline{Q}}|\nabla u| \lesssim \Lambda t
$$

Let us now go back to proving (79) for small $t$. It is enough to prove that the function $t \mapsto \frac{d}{d t} \int_{\underline{Q}} u_{t}$ is Lipschitz continuous at $t=0$, with a Lipschitz constant depending only on the total variation of $u$ (the bound $\Lambda$ from the statement). Indeed, by (88),

$$
\left|\frac{d}{d t} \int_{\underline{Q}} u_{t}-\frac{d}{d t}\right|_{t=0} \int_{\underline{Q}} u_{t}\left|\leq \int_{\underline{Q}}\right| u_{s}-u|| \nabla \cdot \xi\left|\stackrel{\sqrt[809]{\lesssim}}{\lesssim} \int_{\underline{Q}}\right| u_{s}-u \mid .
$$

Hence $t \mapsto \frac{d}{d t}\left|\int_{\underline{Q}} u_{t}\right|$ is Lipschitz continuous at $t=0$ provided the function $t \mapsto \int_{\underline{Q}}\left|u_{t}-u\right|$ is as well. To this purpose, we make use of

$$
\int_{\underline{Q}}\left|u_{s}-u\right|=\sup _{\zeta \in C_{0}^{\infty}(\underline{Q}),|\zeta| \leq 1} \int_{\underline{Q}}\left(u_{s}-u\right) \zeta .
$$

In particular, it suffices to show that the functions $t \mapsto \int_{\underline{Q}}\left(u_{t}-u\right) \zeta$ are uniformly Lipschitz continuous at $t=0$. For this, we note that by a similar argument as for (88), we have

$$
\frac{d}{d t} \int_{\underline{Q}}\left(u_{t}-u\right) \zeta=\frac{d}{d t} \int_{\underline{Q}} u_{t} \zeta=\int_{\underline{Q}} u_{t} \nabla \cdot(\zeta \xi)
$$

and thus

$$
\left|\frac{d}{d t} \int_{\underline{Q}}\left(u_{t}-u\right) \zeta\right| \leq \sup _{\underline{Q}}|\zeta \xi| \int_{\underline{Q}}\left|\nabla u_{t}\right| \stackrel{(90),(14)}{\lesssim \Lambda} \sup _{\underline{Q}}|\xi| \stackrel{\text { (89) }}{\lesssim \Lambda} 1 .
$$

Proof of Lemma 20, Let us choose a smooth cut-off function $\eta$ compactly supported in $Q_{\lambda} \cup \underline{Q_{\lambda}}$ and such that $f_{Q_{\lambda}}(1-\eta) \leq 1 / 4$, while $|\nabla \eta| \lesssim 1 / \lambda$. On the one hand, this implies

$$
\left|f_{\underline{Q_{\lambda}}} u\right| \leq\left|f_{\underline{Q_{\lambda}}} \eta u\right|+\frac{1}{4}
$$

so that it is enough to establish

$$
\left|f_{\underline{Q_{\lambda}}} \eta u\right| \leq \frac{1}{4}
$$


On the other hand we obtain from (2)

$$
\int_{\underline{Q_{\lambda}}} \eta u=-\int_{Q_{\lambda}} \nabla \eta \cdot \mathbf{b}
$$

and as $|\nabla \eta| \lesssim 1 / \lambda$, by the Hölder inequality and Theorem 3, we have

$$
\left|f_{\underline{Q_{\lambda}}} \eta u\right| \lesssim\left(\frac{1}{\lambda^{d}} \int_{Q_{\lambda}}|\mathbf{b}|^{2}\right)^{1 / 2} \lesssim \frac{1}{\lambda^{1 / 2}},
$$

so that the conclusion follows provided $\lambda \gg 1$.

\section{ACKNOWLEDGEMENTS}

We thank Tobias Ried for helpful comments on the manuscript and Emanuele Spadaro for suggesting the reference [14]. A. J. gratefully acknowledges the hospitality of the Max Planck Institute in Leipzig.

\section{REFERENCES}

1. Robert A. Adams and John J. F. Fournier, Sobolev spaces, second ed., Pure and Applied Mathematics (Amsterdam), vol. 140, Elsevier/Academic Press, Amsterdam, 2003.

2. Giovanni Alberti, Rustum Choksi, and Felix Otto, Uniform energy distribution for an isoperimetric problem with long-range interactions, J. Amer. Math. Soc. 22 (2009), no. 2, 569-605.

3. Luigi Ambrosio, Nicola Fusco, and Diego Pallara, Functions of bounded variation and free discontinuity problems, Oxford Science Publications, Clarendon Press, 2000.

4. Scott N. Armstrong and Sylvia Serfaty, Local laws and rigidity for Coulomb gases at any temperature, arXiv e-prints (2019), arXiv:1906.09848.

5. Scott N. Armstrong and Charles K. Smart, Quantitative stochastic homogenization of convex integral functionals, Ann. Sci. Éc. Norm. Supér. (4) 49 (2016), no. 2, 423-481.

6. David P. Bourne, Mark A. Peletier, and Florian Theil, Optimality of the triangular lattice for a particle system with Wasserstein interaction, Comm. Math. Phys. 329 (2014), no. 1, 117-140.

7. Haïm Brézis, Opérateurs maximaux monotones et semi-groupes de contractions dans les espaces de Hilbert., vol. 5, Elsevier, Amsterdam, 1973 (French).

8. Luis Caffarelli and Luis Silvestre, An extension problem related to the fractional Laplacian., Commun. Partial Differ. Equations 32 (2007), no. 8, 1245-1260.

9. Rustum Choksi and Mark A. Peletier, Small volume fraction limit of the diblock copolymer problem: I. Sharp-interface functional, SIAM J. Math. Anal. 42 (2010), no. 3, 1334-1370.

10. Marco Cicalese and Emanuele Spadaro, Droplet minimizers of an isoperimetric problem with longrange interactions, Comm. Pure Appl. Math. 66 (2013), no. 8, 1298-1333.

11. Eleonora Cinti and Felix Otto, Interpolation inequalities in pattern formation., J. Funct. Anal. 271 (2016), no. 11, 3348-3392 (English).

12. Sergio Conti, Branched microstructures: scaling and asymptotic self-similarity, Comm. Pure Appl. Math. 53 (2000), no. 11, 1448-1474.

13. Robert Dautray and Jacques-Louis Lions, Mathematical analysis and numerical methods for science and technology. Vol. 3, Springer-Verlag, Berlin, 1990, Spectral theory and applications, With the collaboration of Michel Artola and Michel Cessenat, Translated from the French by John C. Amson.

14. Luca Esposito and Nicola Fusco, A remark on a free interface problem with volume constraint, J. Convex Anal. 18 (2011), no. 2, 417-426.

15. David Gilbarg and Neil S. Trudinger, Elliptic partial differential equations of second order. Reprint of the 1998 ed., reprint of the 1998 ed. ed., Berlin: Springer, 2001.

16. Alessandro Giuliani and Stefan Müller, Striped periodic minimizers of a two-dimensional model for martensitic phase transitions, Comm. Math. Phys. 309 (2012), no. 2, 313-339. 
17. Enrico Giusti, Minimal surfaces and functions of bounded variation, 1 ed., Monographs in Mathematics, Birkhäuser Boston, 1984.

18. Michael Goldman and Felix Otto, A variational proof of partial regularity for optimal transportation maps, Accepted in Ann. Sci. Éc. Norm. Supér. (2017), arXiv:1704.05339.

19. Hans Knüpfer and Cyrill B. Muratov, On an isoperimetric problem with a competing nonlocal term I: The planar case, Comm. Pure Appl. Math. 66 (2013), no. 7, 1129-1162.

20. Jianfeng Lu and Felix Otto, Nonexistence of a minimizer for Thomas-Fermi-Dirac-von Weizsäcker model, Comm. Pure Appl. Math. 67 (2014), no. 10, 1605-1617.

21. Francesco Maggi, Sets of finite perimeter and geometric variational problems, Cambridge Studies in Advanced Mathematics, vol. 135, Cambridge University Press, Cambridge, 2012, An introduction to geometric measure theory.

22. Tatsuya Miura and Felix Otto, Sharp boundary $\varepsilon$-regularity of optimal transport maps, arXiv e-prints (2020), arXiv:2002.08668.

23. Stefan Müller, Singular perturbations as a selection criterion for periodic minimizing sequences, Calc. Var. Partial Differential Equations 1 (1993), no. 2, 169-204.

24. Felix Otto and Thomas Viehmann, Domain branching in uniaxial ferromagnets: asymptotic behavior of the energy, Calc. Var. Partial Differential Equations 38 (2010), no. 1-2, 135-181.

25. Florian Theil, A proof of crystallization in two dimensions, Comm. Math. Phys. 262 (2006), no. 1, 209-236.

(K. Bellova) Institute of Mathematics, Leipzig University, Augustusplatz 10, 04109 Leipzig, Germany

E-mail address: bellova@math.uni-leipzig.de

(A. Julia) Dipartimento di Matematica "T. Levi-Civita", via Trieste 63, 35121 Padova, ITALY.

E-mail address: ajulia@math.unipd.it

(F. Otto) Max Planck Institute for Mathematics in the Sciences, Inselstrasse 22, 04103 Leipzig, Germany

E-mail address: otto@mis.mpg.de 